\title{
On the identifiability of Anand visco-plastic model parameters using the Virtual Fields Method
}

\author{
S. N. Grama ${ }^{\mathrm{a}}$, S. J. Subramanian ${ }^{\mathrm{a}, *}$, F. Pierron ${ }^{\mathrm{b}}$ \\ ${ }^{a}$ Department of Engineering Design, \\ Indian Institute of Technology, Madras, \\ Chennai-600036, India \\ ${ }^{b}$ Faculty of Engineering and the Environment, \\ University of Southampton, \\ Highfield, Southampton, SO17 1BJ, UK
}

\begin{abstract}
In this paper, the issue of the identification of constitutive parameters of the Anand visco-plastic model is addressed using the Virtual Fields Method (VFM) in an infinitesimal deformation framework. By using VFM, one can take advantage of heterogeneous strain fields obtained by full-field experimental techniques, such as Digital Image Correlation (DIC). Since a wide range of strains and strain rates are sampled in a typical heterogeneous strain field, the number of experiments required to reliably estimate constitutive parameters, especially of rate-dependent materials, is significantly smaller than that needed if conventional experiments (such as uniaxial tension or pure shear configurations) leading to nominally homogeneous strain states were used. However, for such an approach to be successful, the test configuration and loading program should be such that all the constitutive parameters play a significant role (are 'activated') in the resulting strain fields. An analysis of the Anand constitutive model shows that 4 of the 8 parameters can only be found to within a multiplicative constant from full-field kinematic data. Therefore, one of these 4 constants is arbitrarily chosen and the activation of the remaining 7 material parameters is investigated by performing a series of one-element models. Detailed sensitivities of the VFM cost function to these material parameters are derived for a variety of normal stress to shear stress ratios and loading rates. Two main conclusions are drawn based on this one-element study: i) the VFM cost function sensitivities to the material parameters do not vary significantly with loading ratios or rates, and ii)

\footnotetext{
${ }^{*}$ Corresponding author

Email address: shankar_sj@iitm.ac.in (S. J. Subramanian)
} 
2 of the 7 material parameters are not activated for any of the loading ratios or rates considered. Based on the results of the finite-element study, a modified single lap-shear test configuration is designed to yield heterogeneous strains in the joint. Deformation data from a finite-element analysis of this experiment are used as inputs to a VFM routine to compute the Anand material parameters. Our results highlight that non-uniqueness of the identified parameters is a significant issue. The effect of the choice of the cost function and the loading profile on the inverse technique is also thoroughly investigated.

Keywords: Virtual Fields Method; constitutive behavior; elastic-viscoplastic material; numerical algorithms; mechanical testing

\section{Introduction}

Material characterization plays an important role in finite-element modeling of new components or processes in various branches of engineering. The accuracy of the computational model usually depends on the validity of the constitutive model used as well as the reliable measurement or estimation of the parameters used in the material model. The latter task is relatively straightforward for simpler constitutive models such as linear elasticity, but becomes progressively more difficult for complicated models such as those used for rate-dependent plasticity. Various phenomenological and physically motivated constitutive models have been proposed in the literature to describe the combined behavior of rate-independent plasticity and creep effects in the same set of equations $(1 ; ; 2 ; 3 ; 4 ; 5 ; 6 ; 7 ; 8 ; 9 ; 10)$. These equations describe important characteristics related to inelastic deformation including strain rate dependence, isotropic or kinematic hardening, hydrostatic pressure and temperature dependence and evolving micro-structural state of the material $(11 ; 12 ; 13 ; 14 ; 15)$. The material parameters of these constitutive models are usually obtained from simple experiments based on either phenomenological or physical interpretation of the material parameters. The Johnson-Cook model (1), although empirical, is a popular one wherein the functional form of stress includes strain, strain-rate and temperature dependence and is primarily used for modeling hot-working in high strain-rate regime. In overstress-based models (2; 3), the stress can exceed the rate-independent yield surface and relax back to it over time. Physically based material models are usually based on the theory of dislocations $(4,5)$ and crystallographic slip (6; 7) and some of them are shown to be valid over a wide range of strain-rates and temperatures. Another 
class of constitutive models is based on the fact that the intricate physics of plastic deformation is assumed to be captured by only a few internal variables $(8 ; 9 ; 10)$, which are named 'hardness', 'average dislocation density' and 'deformation resistance' in Bodner-Partom (8), Estrin-Mecking (9) and Anand (10; 16) constitutive models respectively. Typically, the internal variables are chosen to be scalars for modeling initially isotropic materials and the evolution of these variables are also specified as part of the constitutive model. A review on the historical use of internal variables in modeling inelasticity is given by Horstemeyer and Bammann (17).

Apart from modeling hot working of metals, one of the commercially important problems that visco-plastic models address is the deformation of solders, which are widely used to provide mechanical or electromechanical connectivity in microelectronics and other branches of engineering. Various rate-dependent constitutive models have been proposed in the literature to describe such deformation $(18 ; 19 ; 20)$. Several studies $(21 ; 22 ;, 23)$ have also shown that the Anand model can be successfully applied to study the deformation behavior of solder alloys; the fact that the Anand visco-plastic model is pre-built in many commercial finite-element softwares including Abaqus ${ }^{\mathrm{TM}}$, Ansys $^{\mathrm{TM}}$ and Adina ${ }^{\mathrm{TM}}$ makes it easier to perform finite-element analysis using this model. Recently, the original Anand model $(10,16)$ has been modified to better describe the behavior of solder joints $(24 ; 25 ; 26)$ and conventional characterization techniques have been used to obtain material parameters.

Conventional material characterization relies on experiments which yield nominally homogeneous strain and stress states from which material parameters are obtained through curve fitting. For instance, Kowalewski et al. (27) performed creep tests on an $\mathrm{Al}$ alloy at $150^{\circ} \mathrm{C}$ at various stress levels and defined a cost function based on the sum of squared differences between experimental and fitted strain-time curves:

$$
\phi_{0}=\sum_{i=1}^{N_{\mathrm{t}}}\left[\left(\sum_{j=1}^{N_{\mathrm{e}}}\left(\varepsilon^{\mathrm{f}}-\varepsilon^{\exp }\right)^{2}+W_{i}\left(t_{i}^{\mathrm{f}}-t_{i}^{\exp }\right) / t_{i}^{\exp }\right)\right],
$$

where $\varepsilon^{\mathrm{f}}$ and $\varepsilon^{\exp }$ correspond to fitted and experimentally computed strains respectively, $W_{i}$ are weighting factors, $t^{\mathrm{f}}$ and $t^{\mathrm{exp}}$ correspond to predicted and experimental lifetimes, and $N_{\mathrm{t}}$ and $N_{\mathrm{e}}$ refer to the number of creep curves and the number of points per curve respectively. This cost function was minimized to yield the material parameters. However, this procedure has limitations in 
ensuring the best quality of fit as it fails to accommodate for different scales of strain and time and fails to consider the contributions of all the points of all the curves, especially if multiple curves need to be fitted as shown by $\mathrm{Li}$ et al. (28). To avoid such limitations and ensure robustness in the identification procedure, the objective function has been reformulated in other works $(29$; 30$)$. Another inherent drawback of the conventional approach to material characterization is the large number of experiments required to encompass a sufficiently wide range of strain rates and temperatures, which becomes important especially for visco-plastic materials. Finally, in conventional material characterization, the assumption of homogeneous strain and stress states, which allows for easy interpretation of the experimental data, is violated at large deformations (e.g. due to necking in uni-axial tension). This necessitates the design of other test configurations which can give rise to large strain levels without strain localization (31) or modifications in the formulation of the inverse procedure (32).

A recent alternative to circumvent these limitations is the use of experiments that lead to nominally heterogeneous strain states, which are measured through full-field experimental techniques such as Digital Image Correlation (DIC), Moiré interferometry, grid method, etc. and later processed with a suitable full-field inverse technique such as the Constitutive Equation Gap Method (CEGM, (33; 34)) and its variants, the Constitutive Compatibility Method (CCM, (35)) and the Dissipative Gap method (DGM, (36)); the Equilibrium Gap Method (EGM, (37)), the Self-Optimizing Method (SOM, (38)), the Finite-Element Updating Method (FEMU, (39)) and the Virtual Fields Method (VFM, (40; 41)) and its variants Eigenfunction VFM (EVFM, (42, 43)), Fourier-VFM (44). An overview of these identification techniques is presented in (45). In these methods, experiments leading to heterogeneous states of strain are employed and a broad range of strains and strain-rates are typically sampled in a single test; and as every measured data point participates in the optimization technique, more constraints are implicitly imposed on the cost function to be minimized, thereby ensuring that the computed material parameters are applicable over a wide range of strains and strain rates. In CEGM, the focus is to obtain an admissible stress field through the minimization of constitutive equation error over the kinematically admissible displacement and thermodynamically admissible material parameter space; the CCM reduces the computational cost of CEGM by decoupling the identification of stress from the identification of material parameters, while DGM relies on the error in dissipation for elasto-plastic material identification; EGM makes 
use of the equilibrium deviation between neighboring elements in a discretized domain for material parameter and hence, damage identification; and SOM requires traction and displacement information on the boundary and estimates the material parameters through the minimization of virtual work integrals obtained from two parallel FE simulations of displacement and traction boundary conditions respectively. On the other hand, in FEMU, a finite element model of the actual test configuration is built up and the material parameters are iteratively tuned by repeatedly performing finite-element analyses until a close correspondence between experimental and numerical field variables is achieved. Depending on the choice of field variables, the technique is either called FEMU-F (force) or FEMU-U (displacement). Although these techniques are quite popular, they incur high computational expense due to the large number of finite-element analyses required.

Of late, VFM has been receiving increased attention due to the direct nature of material parameter estimation used herein. VFM is derived from the principle of virtual work, which is a statement of equations of equilibrium in weak form (46). When body forces are absent, the principle of virtual work under the assumption of static loading and small deformation framework can be written as (47):

$$
\int_{S} \boldsymbol{t} \cdot \boldsymbol{u}^{*} d S=\int_{V} \boldsymbol{\sigma}: \boldsymbol{\varepsilon}^{*} d V,
$$

where $\boldsymbol{t}$ represents the actual traction at the boundary of the considered volume, $\boldsymbol{u}^{*}$ represents any differentiable virtual displacement field, $\boldsymbol{\sigma}$ represents the actual stress field and $\boldsymbol{\varepsilon}^{*}$ the virtual strain field obtained by differentiation of the virtual displacement field. The actual stress $\sigma$ can be expressed in terms of the actual strains using the constitutive equations. Thus Eqn. (2) can be rewritten in terms of the unknown material parameters, known actual tractions, actual strains and chosen virtual fields. By making an appropriate choice for the virtual displacement field in Eqn. (2), one equation for the set of unknown material parameters is generated at each deformation step. As full-field kinematic data are typically available at many deformation steps, an over-determined system of equations for the unknown material parameters is generated, which is solved in a least squares sense until the right hand side of Eqn. (2) (the 'internal virtual work') closely matches the left hand side (the 'external virtual work'). For linear constitutive equations, the resulting system of equations is linear $(48,43)$, whereas for nonlinear constitutive models, the resulting system is 


\footnotetext{
${ }^{1}$ This is not always the case; e.g. for some simple hyper-elastic constitutive models, which are non-linear in the constitutive parameters, the resulting system of equations is linear.
}

Merely obtaining heterogeneous strain fields from an experiment is not sufficient to ensure accurate computation of all the material parameters; unless the material parameter is strongly activated (i.e. has a strong influence on the measured kinematic fields), it cannot be uniquely ascertained using any inverse scheme. In order to ensure such activation, optimization of the geometry of the specimen and loading profiles is often performed. This approach directly affects the wellposedness of the inverse problem and is an active area of research. Pierron et al. (48) optimized the free length and the orthotropic axis angle of the unnotched Iosipescu specimen to extract the orthotropic material parameters and more recently, Wang et al. (49) achieved the same for a foam material. Robert et al. (50) qualitatively compared the experimental configurations of Haddadi and Belhabib (51) and Meuwissen et al. (52), which are used for elasto-plastic material characterization. A methodology for the design of test configuration considering all the errors in the identification chain has been recently proposed by Rossi and Pierron (53). The loading protocol used in the experiment also plays an important role in inverse problems: Pagnotta (54) and Bruno et al. (55) optimized the loading profile for retrieving elastic material parameters reliably. In general, refinement of the experiment is done in order to ensure strain and strain-rate heterogeneity and thus ensure activation of the material parameters.

Many researchers also perform refinement of the objective function to ensure that uncertainties in the experimental data are suitably accounted for. For instance, Meuwissen et al. (52) and Mathieu et al. (56) assigned higher weights to larger strain values than to smaller strains in their objective function as it is well known that the uncertainty in strains computed using DIC is much higher for smaller strain levels when compared with finite strains $($ 57, 58). Even if all material parameters are activated and experimental uncertainties are suitably accounted for in the objective function, the minimization algorithm may be trapped in any of a number of local minima due to the cost function being highly non-linear. In order to avoid this issue, some researchers $(59 ; 60)$ have explored the idea of using evolutionary algorithms at the expense of computational efficiency.

In the present work, a systematic approach for evaluating material parameters of the Anand model using full-field data and the Virtual Fields Method is described. Although the VFM has

typically non-linear 1 and is usually solved as a minimization problem. 
been previously applied to non-linear material characterization, see (61) for instance, to the best of the authors' knowledge, this is the first application of the VFM to an inelastic model with more than 4 material parameters. The emphasis in this work is to demonstrate the feasibility of our approach in estimating the material parameters of this challenging inelastic model with 8 parameters. Therefore, kinematic data generated synthetically using finite element analysis of a new test specimen designed to activate a number of the relevant material constants is used in lieu of actual experimental data. By adopting this approach, errors due to the choice of an inappropriate material model or experimental noise is avoided. The application of the proposed methodology to experimental data will be pursued elsewhere, as will a comparison of the results obtained with the proposed methodology using conventional VFM with those using the related Eigenfunction Virtual Fields Method (42; 43). The rest of the paper is organized as follows: in Section 2, a brief outline of the Anand visco-plastic model and an identifiability issue is presented; in Section 3, a procedure of refinement of the test configuration through a sensitivity analysis of a one-element model with varying normal to shear-stress ratios is described; in Section 4, a lap-shear configuration is designed using the results of the sensitivity analysis and the issue of identifiability of material parameters is investigated using different load profiles and finally, a few concluding remarks are offered in Section 5 .

\section{The Anand model and an identifiability issue}

The Anand visco-plastic model (10) is an internal variable based model in which rate-dependent and rate-independent plasticity effects are unified. The model employs a single scalar internal variable, $s$, which represents the isotropic resistance to macroscopic plastic flow. The model does not have an explicit yield criterion or a loading-unloading criterion and visco-plastic flow occurs for any non-zero stress.

Motivated by experiments on $\mathrm{Al}$ and $\mathrm{Fe}-2 \% \mathrm{Si}$, Brown et al. (16) proposed the following functional form for the flow equation, which includes both power-law and exponential dependence of strain rate on stress:

$$
\dot{\tilde{\varepsilon}}^{\mathrm{p}}=f(q, s, \theta)=A \exp \left(\frac{-Q}{R \theta}\right)\left[\sinh \left(\xi \frac{q}{s}\right)\right]^{1 / m}
$$

where $\dot{\tilde{\varepsilon}}^{\mathrm{p}}$ is the equivalent plastic strain rate, $A$ is a pre-exponential factor, $Q$ is the activation energy, 
$R$ is the universal gas constant, $\xi$ is a multiplier of stress, $\theta$ is temperature and $q$ is the von Mises stress. The flow equation is complemented by an evolution equation for the internal variable $s$ :

$$
\dot{s}=g(q, s, \theta) \dot{\tilde{\varepsilon}}^{\mathrm{p}}=\left[h_{0}\left|1-\frac{s}{s^{*}}\right|^{a} \operatorname{sgn}\left(1-\frac{s}{s^{*}}\right)\right] \dot{\tilde{\varepsilon}}^{\mathrm{p}},
$$

where $h_{0}$ represents hardening, $a$ represents strain rate sensitivity of hardening and $s^{*}$ represents a saturation value of deformation resistance at a given strain rate $\dot{\tilde{\varepsilon}}^{p}$ and temperature $\theta$ given by

$$
s^{*}=\tilde{s}\left[\frac{\dot{\tilde{\varepsilon}}^{\mathrm{p}}}{A} \exp \left(\frac{Q}{R \theta}\right)\right]^{n}=\tilde{s}\left[\sinh \left(\xi \frac{q}{s}\right)\right]^{n / m},
$$

where, $n$ represents the strain rate sensitivity of deformation resistance and $\tilde{s}$ is a material parameter. The signum term is added to accommodate for situations when $s>s^{*}$, e.g. during rapid reduction in strain-rate or rapid rise in temperature and this term also models strain softening situations. However, for general loading situations in which such rapid strain-rate or temperature changes are not encountered, it can be assumed that $s \leq s^{*}$ in the Anand model.

In the present work, the focus is on isothermal deformation, therefore it is not possible to obtain material parameters $Q$ and $A$ separately, instead they are combined and retrieved as a single parameter $C=A \exp \left(\frac{-Q}{R \theta}\right)$. From Eqn. 3 , it can be seen that when plastic flow is fully established $\left(\dot{\tilde{\varepsilon}} \approx \dot{\tilde{\varepsilon}}^{\mathrm{p}}\right)$, the applied stress is directly proportional to $s$ :

$$
q=\frac{1}{\xi} \sinh ^{-1}\left[\left(\frac{\dot{\tilde{\varepsilon}}^{\mathrm{p}}}{A} \exp \frac{Q}{R \theta}\right)^{m}\right] s
$$

This approximation has been used previously to identify the Anand model parameters (16). However, the objective of the present work is to recover the constitutive parameters $C, m, n, a, \tilde{s}, h_{0}, s_{0}$ and $\xi$ from full-field kinematic data. An interesting issue of identifiability of the four parameters $\tilde{s}, h_{0}, s_{0}$ and $\xi$ arises from the nature of the Anand constitutive model (Eqns. 3 and 4 ). If the parameters $C, m, n$ and $a$ are held at their true values, while $\tilde{s}, h_{0}, s_{0}$ and $\xi$ are scaled from their true values by an arbitrary multiplicative constant $\alpha$, then an analysis of Eqns. (3) and (4) shows that for a given $\dot{\tilde{\varepsilon}}^{p}$, one obtains the same stress, irrespective of the value of $\alpha$. Thus, even in principle, knowledge of full-field kinematic variables and the total load is sufficient to estimate the actual values of the four parameters $\tilde{s}, h_{0}, s_{0}$ and $\xi$ to only within a multiplicative constant. This identifi- 
ability problem is temporarily circumvented in this work by arbitrarily fixing the value of $\xi$ to be 7, a value previously obtained for eutectic SnAg solder (24); thus, a functional form similar to a variant of the Anand model, proposed in $(\underline{62})$ is recovered. This observation will have a significant bearing on the identifiability of the other Anand model parameters as discussed in Section 4.

\section{Optimization of test configuration}

The issue of choosing the test configuration plays a pivotal role in the inverse problem. For example, direct determination of shear modulus of a linear-elastic material from a homogeneous equi-biaxial experiment is not possible. As a prelude to designing a planar test configuration which leads to a balanced activation of all material parameters of the Anand model, a series of infinitesimal deformation one-element models is analyzed for a range of strain rates and normal to shear-stress ratios (Fig. 11). The boundary conditions for this element are chosen so that both normal-stress and shear-stress can be independently varied. The Anand visco-plastic model implemented in Abaqus $^{\mathrm{TM}}$ is utilized in this analysis. Representative Anand model parameters obtained from Chen et al. (24) (Table 1), along with the elastic material parameters $(E=48 \mathrm{GPa}$ and $v=0.36)$ are assigned to the single square element of side $5 \mathrm{~mm}$. The element is assumed to be in a state of plane stress, just as an actual specimen will be, in order to enable computation of the virtual work integrals.

\begin{tabular}{ll}
\hline Material Parameter & Value \\
\hline$C\left(\mathrm{~s}^{-1}\right)$ & $1.624 \times 10^{-9}$ \\
$\tilde{s}(\mathrm{MPa})$ & 52.4 \\
$\xi$ & 7 \\
$m$ & 0.207 \\
$n$ & 0.018 \\
$a$ & 1.6 \\
$h_{0}(\mathrm{MPa})$ & $1.178 \times 10^{5}$ \\
$s_{0}(\mathrm{MPa})$ & 7.198 \\
\hline
\end{tabular}

Table 1: Representative material parameters of Anand model from Chen et al. (24).

Six different loading cases are considered, as shown in Table 2 to span a range of stress states ranging from simple shear to pure uniaxial tension. The loading profiles (Fig. 2) include a linear ramp as well as creep portions, each for 3000 seconds. The displacement and strain histories are 


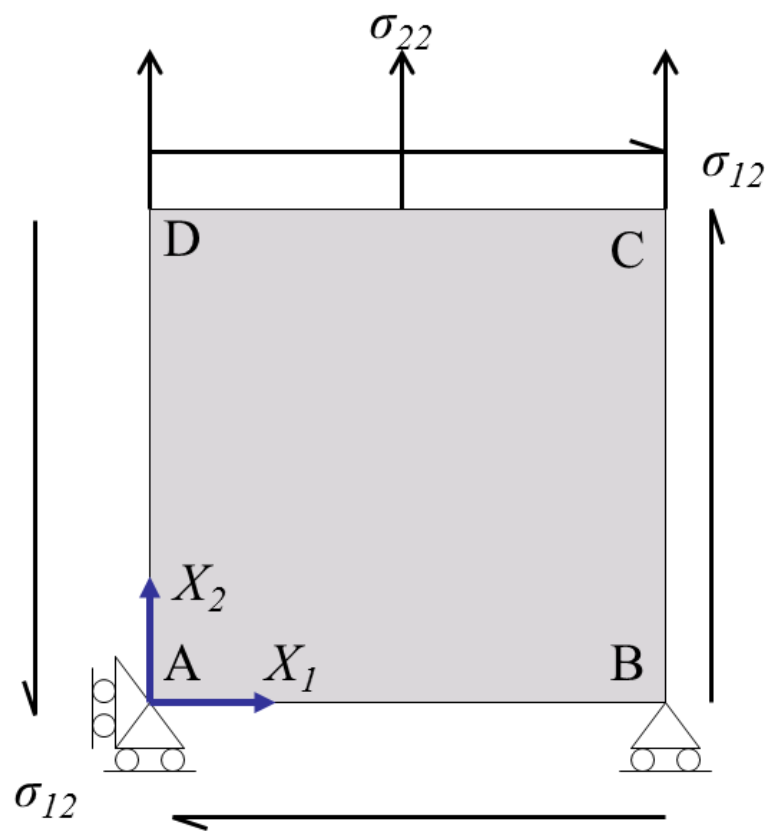

Figure 1: A representative one-element model with the applied boundary conditions, such that the normal stress $\sigma_{22}$ and the shear stress $\sigma_{12}$ can be independently varied. A linear shape function is chosen for the plane stress element, which is a square of side $5 \mathrm{~mm}$.

obtained from the FE analysis and stored, to simulate the experimental data obtained from a fullfield technique. For each of these models, virtual displacement fields are chosen to mimic the true strain fields (Table 2), i.e., either uniform virtual tension, shear or both.

\begin{tabular}{lllll}
\hline Case & $\sigma_{12}(\mathrm{MPa})$ & $\sigma_{22}(\mathrm{MPa})$ & $u_{1}^{*}$ & $u_{2}^{*}$ \\
\hline A & 12 & 0 & $X_{2}$ & 0 \\
B & 11.4 & 3.7 & $X_{2}$ & $X_{2}$ \\
C & 9.7 & 7 & $X_{2}$ & $X_{2}$ \\
D & 7 & 9.7 & $X_{2}$ & $X_{2}$ \\
E & 3.7 & 11.4 & $X_{2}$ & $X_{2}$ \\
F & 0 & 12 & 0 & $X_{2}$ \\
\hline
\end{tabular}

Table 2: Normal-stress to shear-stress ratios ranging from pure normal stress to simple shear are chosen in the finite-element simulation and the virtual fields, $u_{1}^{*}$ and $u_{2}^{*}$ are chosen so as to include all non-zero stress components in the computation of internal virtual work. $X_{2}$ is an independent variable varying from 0 to $5 \mathrm{~mm}$.

As this one-element analysis is stress controlled, the external virtual work is calculated straight- 
forwardly in terms of the applied tractions:

$$
W_{\mathrm{ext}}^{*}=\int_{C D}\left(t_{1} u_{1}^{*}+t_{2} u_{2}^{*}\right) d X_{1}+\int_{D A} t_{2} u_{2}^{*} d X_{2}+\int_{B C} t_{2} u_{2}^{*} d X_{2}
$$

For instance, for loading case $\mathrm{A}$, the only non-zero contribution to $W_{\mathrm{ext}}^{*}$ comes from $t_{1} u_{1}^{*}$ on CD and is equal to $(12 \mathrm{MPa} \times 5 \mathrm{~mm} \times 5 \mathrm{~mm})=0.3 \mathrm{~J}$ per unit thickness. Similarly, for loading case $\mathrm{F}$, $W_{\text {ext }}^{*}$ is again equal to $0.3 \mathrm{~J}$ per unit thickness. The internal virtual work at a particular time step is calculated as

$$
W_{\text {int }}^{*}=\int_{V} \sigma_{i j} \varepsilon_{i j}^{*} d V=\sigma_{i j} \varepsilon_{i j}^{*} A^{\mathrm{e}}
$$

where the thickness is again assumed to be unity and $A^{\mathrm{e}}$ is the area of the element. In order to compute the Cauchy stress $\sigma_{i j}$ from the strains as one would need to do in an experiment, a finite deformation time integration routine based on the one presented in (63) is used. However, since that algorithm is valid only for plane strain and three-dimensional elements, a modified version suitable for plane stress situations is implemented through nested iterations at the integration point level (64), as detailed in Appendix A. Although the internal virtual work integral can be straightforwardly computed from the stresses obtained from FE solution for the present one-element case, this integration scheme will be required to implement the VFM for actual kinematic measurements and therefore, it is developed and used for the one-element case as well.
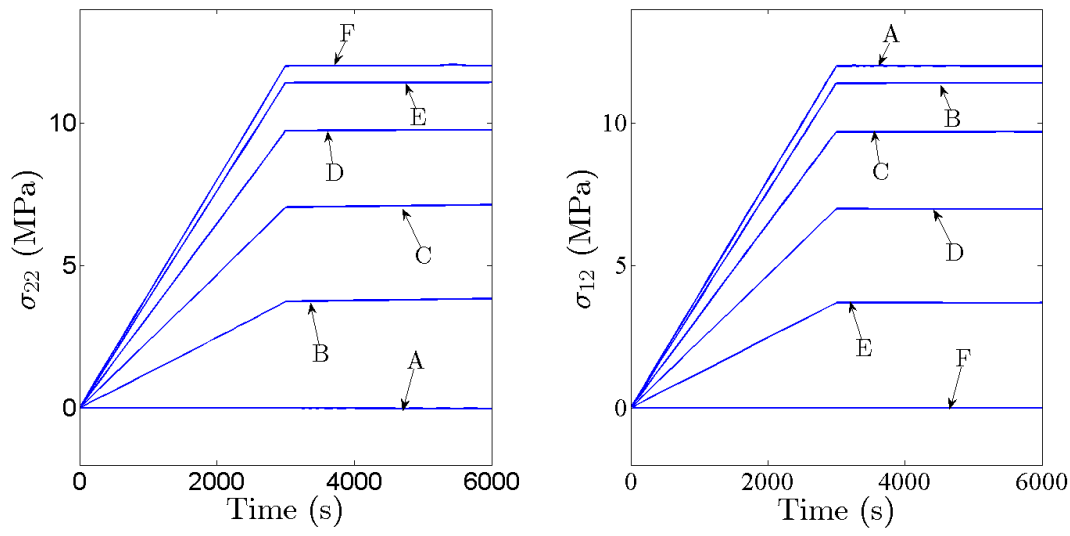

Figure 2: Loading profiles for all test cases include a monotonic loading region as well as creep region.

Once the stresses are computed, the internal virtual work of Eqn. (8) is calculated for each 
time step considered and a cost function $\phi_{1}$ is defined as the normalized sum of squared differences between the internal and external virtual work over all time steps:

$$
\phi_{1}(\boldsymbol{p})=\sum_{1}^{N_{t}}\left[\frac{W_{\mathrm{ext}}^{*}-W_{\mathrm{int}}^{*}(\boldsymbol{p})}{W_{\mathrm{ext}}^{*}}\right]^{2}
$$

where $N_{t}$ represents the number of time steps. One expects that the true material parameter vector $p^{\text {tr }}$ renders the difference between the internal and external work minimal; therefore, the objective is to find the true set of material parameters $\boldsymbol{p}^{\text {tr }}$ by minimizing $\phi_{1}$ with respect to $\boldsymbol{p}$ :

$$
p^{\mathrm{tr}}=\underset{p}{\arg \min }\left[\phi_{1}(\boldsymbol{p})\right]
$$

The cost function $\phi_{1}$ is minimized using the Matlab built-in function fminsearch (based on the Nelder-Mead algorithm) for all the loading scenarios. The chosen guess parameter set converges to the true set for every profile, suggesting that any profile (Table 2) can be used for model parameter identification. To get a more quantitative comparison across the loading cases, $\phi_{1}$ variation with respect to deviation in material parameters from their true values is studied. Figure 3 illustrates the variation of $\phi_{1}$ for the simple-shear loading profile A, when material parameters $n$ and $m$ are varied from their true values by $\pm 10 \%$, while the other material parameters are kept at their true values. Evidently, the minimum is found at the true values of the material parameters, $m$ and $n$ as indicated by vertical and horizontal lines respectively (Fig. 3). The valley formed by $\phi_{1}$ in $m-n$ space is aligned nearly parallel to the $n$ axis indicating the low identifiability of $n$. Further, the slope of $\phi_{1}$ along the valley is very small, indicating that several $(m, n)$ pairs provide similar $\phi_{1}$ values. In order to systematically obtain the relative sensitivities of $\phi_{1}$ to all 8 material parameters, a full-factorial computation is performed over the material parameters at five levels (true values, $\pm 25 \%, \pm 50 \%$ ) using the kinematic data obtained from the finite-element analysis corresponding to the true material parameter set. The normalized sensitivity matrix $\left[\phi_{i j}^{\prime \prime}=\left(\partial^{2} \phi_{1} / \partial p_{i} \partial p_{j}\right) / \min \left(\partial^{2} \phi_{1} / \partial p_{i} \partial p_{j}\right)\right]$ is computed for each loading case, the normalization factor chosen to be the same so that relative sensitivities can be unambiguously compared and the smallest $\phi_{i j}^{\prime \prime}$ is unity. The $\phi_{i j}^{\prime \prime}$ for simple shear at a strain rate of $2 \times 10^{-4} \mathrm{~s}^{-1}$ (Table 3 ) strongly suggests that $\phi_{1}$ is not very sensitive to the parameters $h_{0}, n, C$ and $s_{0}$ (indicated by italic font) whereas $\phi_{1}$ is much more sensitive to parameters $m, \xi$ and $\tilde{s}$ (indicated by bold font), while the sensitivity to $a$ is between those of these two groups 
of material parameters. The same trend is observed across all normal to shear stress ratios; the $\phi_{i j}^{\prime \prime}$ for loading profile $\mathrm{D}$ with $\left(\sigma_{12} / \sigma_{22}=0.72\right)$ is shown in Table 4 .

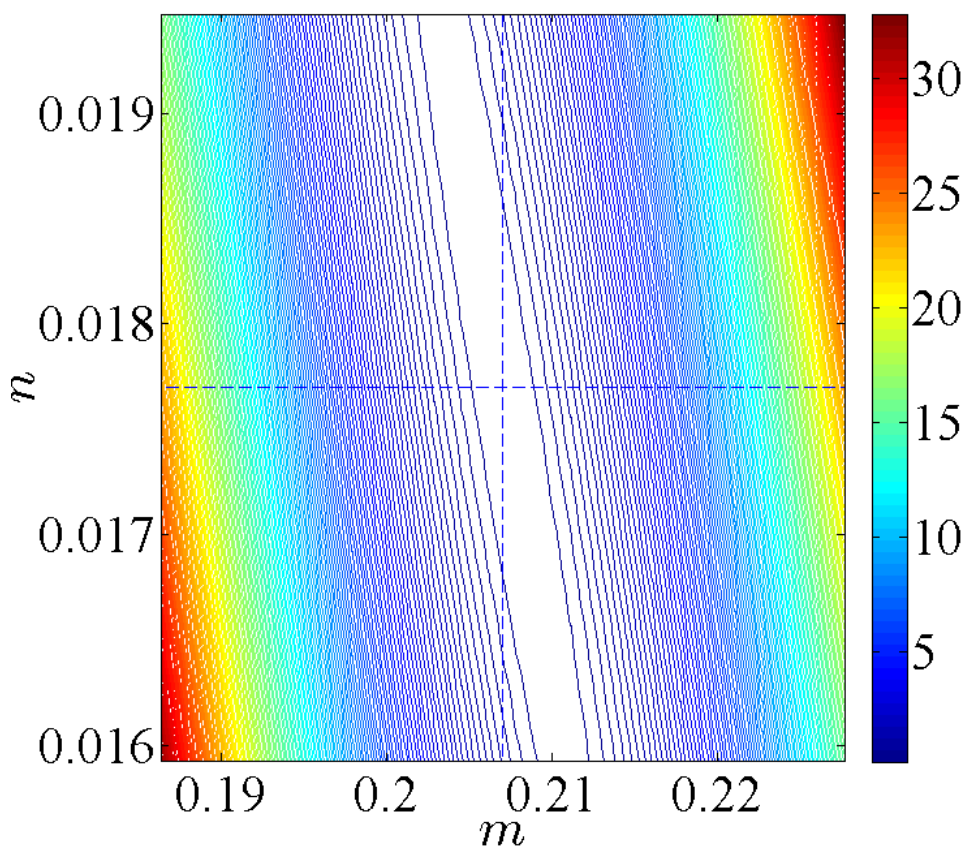

Figure 3: Cost function variation over a range of $n$ and $m$ show a minimum at their true values as indicated by intersection of the horizontal and vertical lines respectively.

The variation in sensitivities with changes in strain-rates (from $10^{-5} \mathrm{~s}^{-1}$ to $10^{-1} \mathrm{~s}^{-1}$ ) is also studied for the simple-shear loading case A. The normalized sensitivity matrix (Table 5) for this loading at a strain rate of $10^{-1} \mathrm{~s}^{-1}$ is similar to that at a strain rate of $2 \times 10^{-4} \mathrm{~s}^{-1}$ (Table 3 ) but with more balanced sensitivities with respect to material parameters $\tilde{s}, m$ and $\xi$.

In order to compare normalized sensitivity matrices across different loading cases, the sensitivity of $\phi_{1}$ with respect to each parameter is normalized by that with respect to $C$. The normalized sensitivity quotients, $\phi_{i i}^{\prime \prime} / \phi_{C C}^{\prime \prime}$ (no summation implied) for various material parameters are shown in Fig. 4. Parameters $C, s_{0}, h_{0}, n$ and $a$ have less influence on $\phi_{1}$ compared to the other parameters $\tilde{s}, m$ and $\xi$. Moreover, across different loading scenarios and applied strain-rates, parameters $C$ and $s_{0}$ consistently have the least impact on $\phi_{1}$ and therefore, it is expected that they will not be uniquely identified by the present approach. Material parameter $C=A \exp (-Q / R \theta)$ has a low impact on the cost function since it does not play a role in the determination of the saturation value of de- 


\begin{tabular}{|c|c|c|c|c|c|c|c|c|}
\hline & $C$ & $\tilde{S}$ & $n$ & $h_{0}$ & $s_{0}$ & $m$ & $a$ & $\xi$ \\
\hline$C$ & 12.4 & 118.2 & 16.4 & 15.7 & 4.1 & 93.9 & 27.9 & 129.9 \\
\hline$\tilde{s}$ & & 1047 & 147.9 & 99.1 & 9.7 & 800.1 & 219.2 & 1085.2 \\
\hline$n$ & & & 21 & 12.2 & 1 & 111.7 & 29 & 150.7 \\
\hline$h_{0}$ & & & & 31.3 & 11.8 & 95.4 & 43.3 & 140.7 \\
\hline$s_{0}$ & & & & & 14.1 & 20.6 & 6.8 & 36.2 \\
\hline$m$ & & sym & & & & 631.5 & 181.2 & 860.1 \\
\hline$a$ & & & & & & & 74.1 & 253.4 \\
\hline$\xi$ & & & & & & & & 626.8 \\
\hline
\end{tabular}

Table 3: The normalized sensitivity matrix for simple-shear loading case A shows that $\phi_{1}$ is not sensitive to parameters $h_{0}, n, C$ and $s_{0}$ (italics) but very sensitive to parameters $m, \xi$ and $\tilde{s}$ (bold).

formation resistance, $s^{*}$ (Eqn. 5), which dictates the value of deformation resistance and hence the stress $(q<s)$. The low sensitivity of $s_{0}$ can be reasoned through the following argument. The initial value of deformation resistance, $s_{0}$, is seen to influence the kinematics of the problem only during the initial phase of deformation and for the various material parameter combinations and strain rates considered here, $s$ saturates quickly irrespective of $s_{0}$ (Figs. 5 and 6). The equivalent stress $q$ profiles corresponding to different $s_{0}$ also differ only in the initial stages of deformation, while they match closely in the creep regime (Fig. 77). Since the cost function $\phi_{1}$ accommodates the contribution of all time steps, the effect of the initial stages of deformation on $\phi_{1}$, and hence of $s_{0}$, is very small. At first sight, it might seem that the identifiability of parameter $s_{0}$ can be improved by only considering the experimental kinematic data from a first few load steps, however the identifiability problem will be made more acute by the presence of higher experimental noise in this regime (typically in DIC), which leads to uncertainties in $s_{0}$ that will render its estimate practically meaningless.

One of the commercially important applications where the present methodology can be applied is the mechanical characterization of solders, which often undergo shear dominated loading largely caused by mismatches in coefficients of thermal expansion. Therefore, a modified lap-shear configuration with the solder joint sandwiched between two rectangular copper substrates (Fig. 8) was designed, which mimics the stress state experienced by typical solder joints. Since the single- 


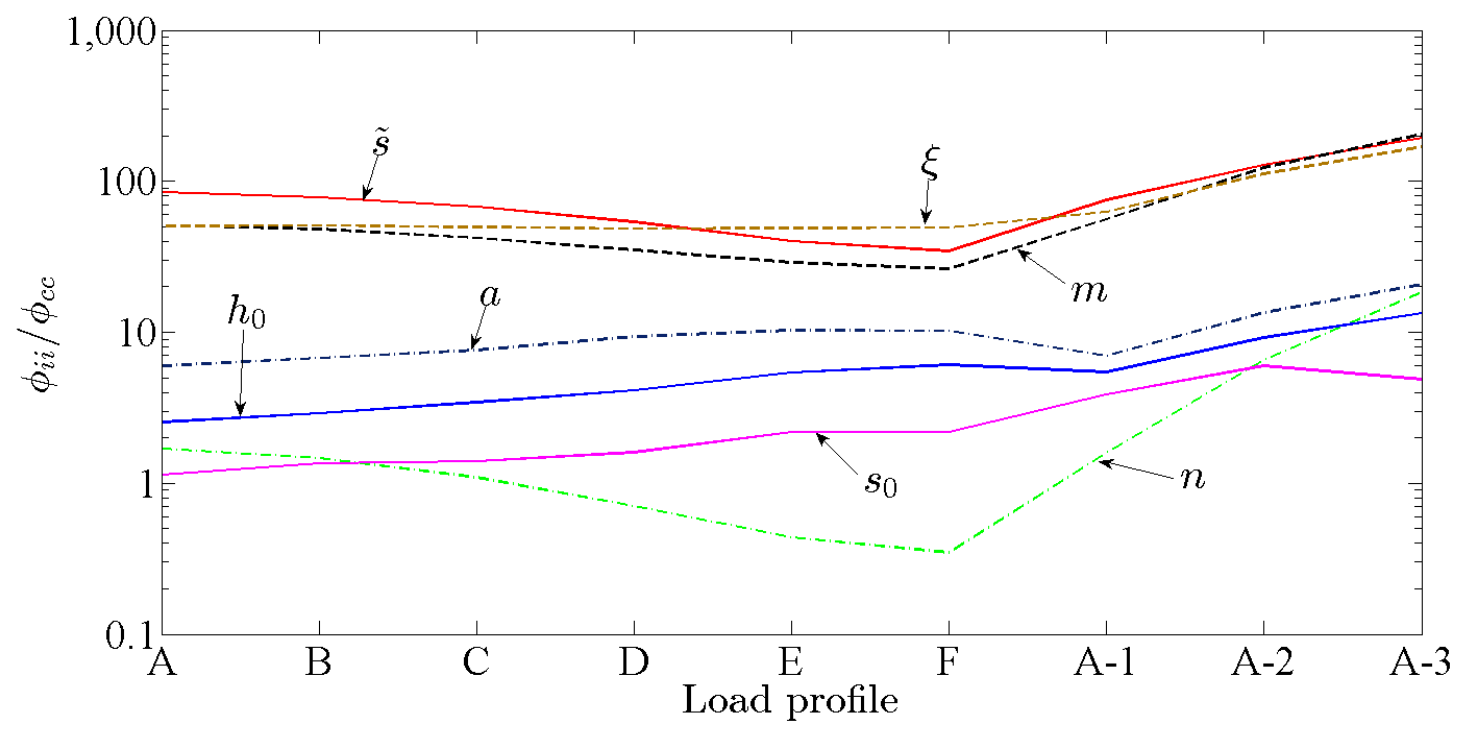

Figure 4: The effect of material parameters on $\phi_{1}$ reveals that parameters $\left(C, s_{0}, h_{0}, n\right.$ and $\left.a\right)$ have less influence, while parameters $(m, \tilde{s}$ and $\xi)$ have a greater influence for an applied strain-rate of $1.6 \times 10^{-5} \mathrm{~s}^{-1}$. The load profiles A-1, A-2 and A-3 correspond to loading profile $A$ at applied strain rates of $10^{-5} \mathrm{~s}^{-1}, 10^{-3} \mathrm{~s}^{-1}$ and $10^{-1} \mathrm{~s}^{-1}$ respectively.

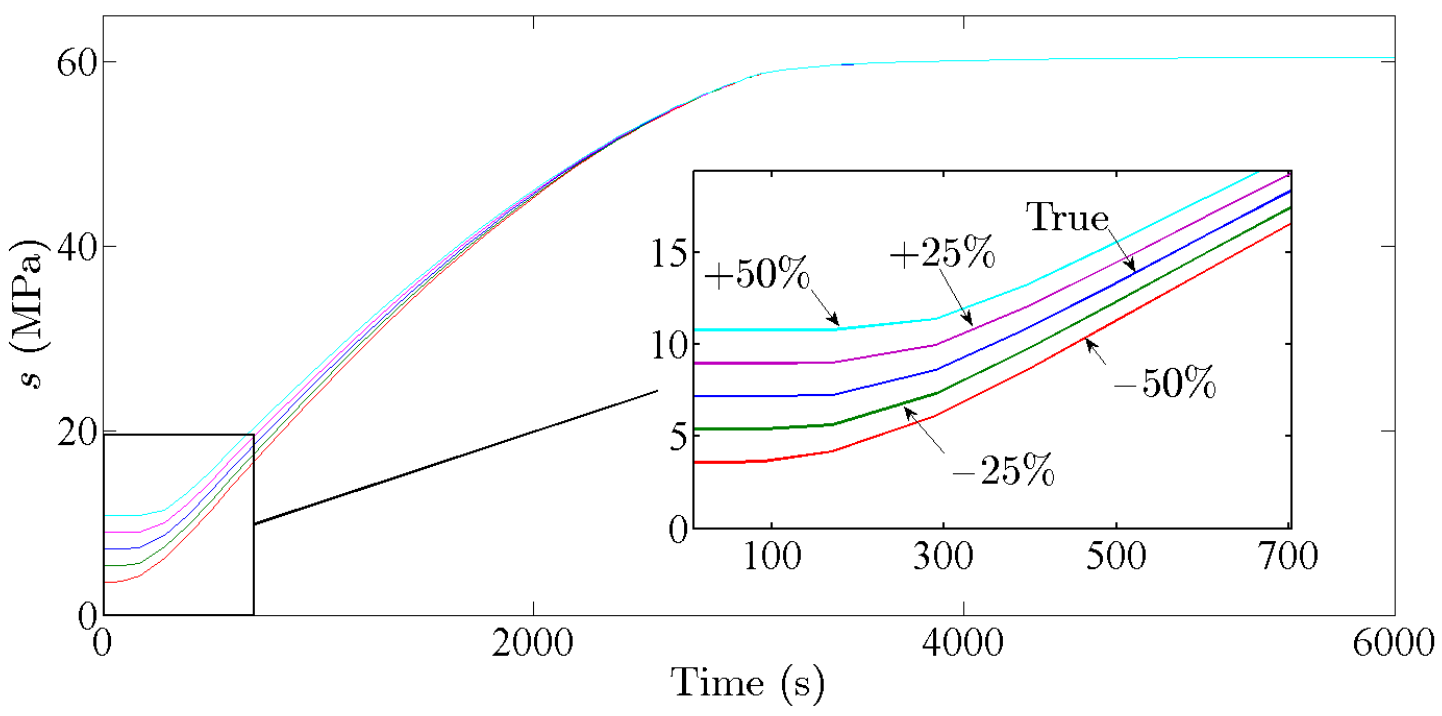

Figure 5: The evolution of the deformation resistance $s$ for loading case A (strain rate of $2 \times 10^{-4}$ $\mathrm{s}^{-1}$ ) corresponding to different initial values of deformation resistance $s_{0}$ indicates observable differences only in the initial stages of deformation. 


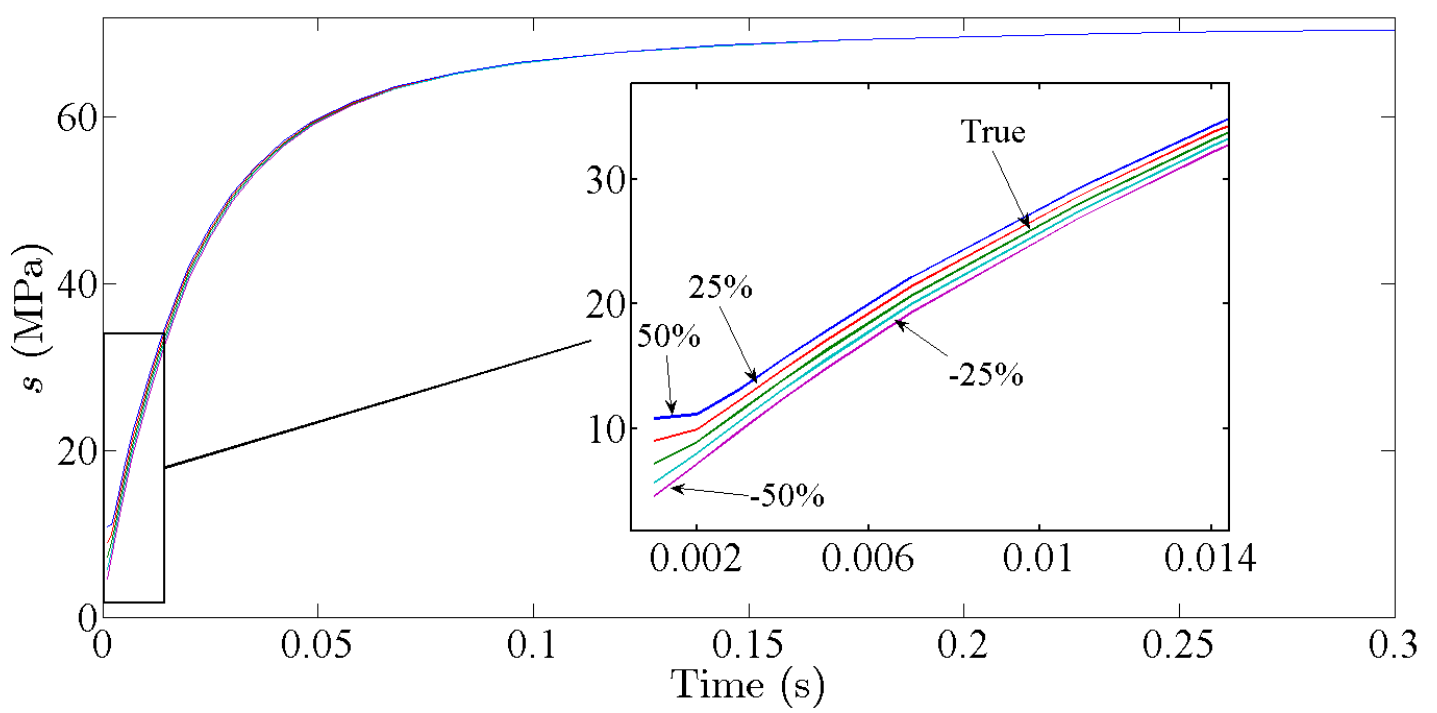

Figure 6: The evolution of deformation resistance $s$ for loading case A at applied strain rate of $10^{-1} \mathrm{~s}^{-1}$ is the same irrespective of $s_{0}$.

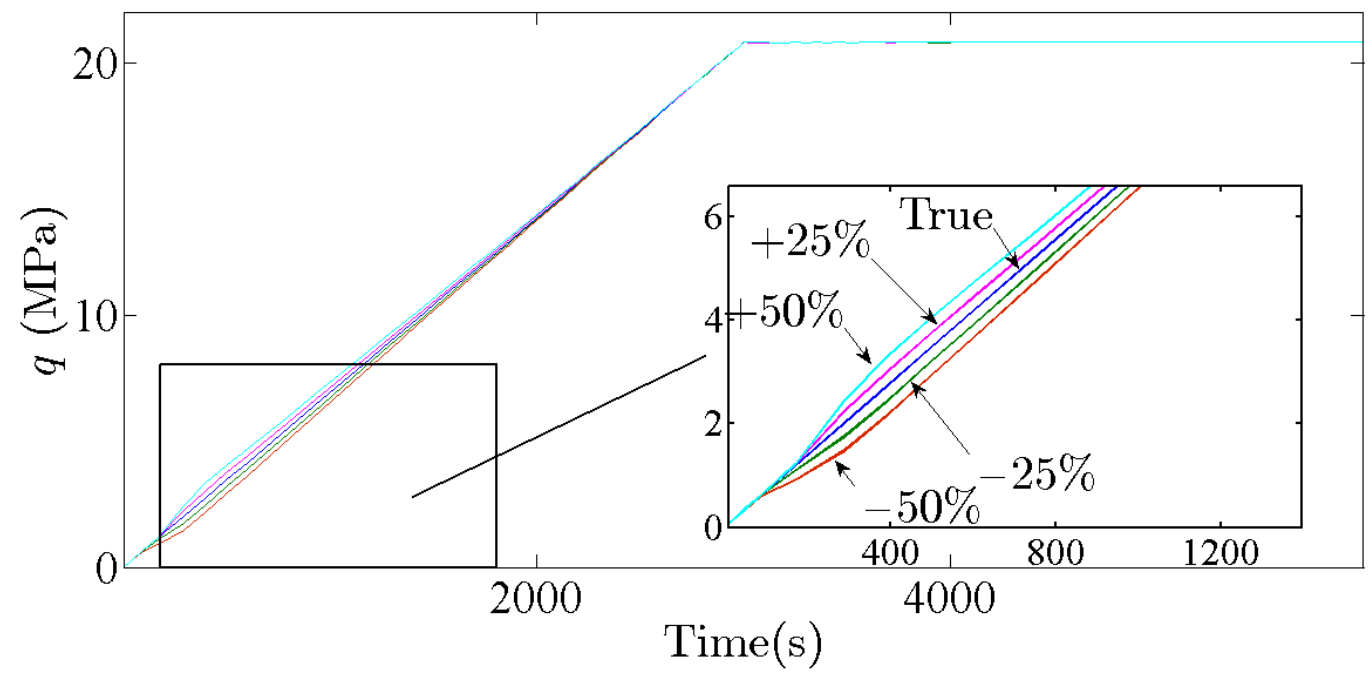

Figure 7: The evolution of equivalent stress $q$ for loading case A (strain rate of $2 \times 10^{-4} \mathrm{~s}^{-1}$ ) indicates observable variation only in the initial stages of deformation (magnified plot) for different $s_{0}$. 


\begin{tabular}{l|llllllll} 
& $C$ & $\tilde{s}$ & $n$ & $h_{0}$ & $s_{0}$ & $m$ & $a$ & $\xi$ \\
\hline$C$ & 7.9 & 60.7 & 6.8 & 16.1 & 4 & 50.1 & 24.9 & 77.7 \\
$\tilde{s}$ & & $\mathbf{4 2 8 . 4}$ & 49.3 & 93.5 & 10.1 & 337.1 & 168.4 & 516 \\
$n$ & & & 5.6 & 10.3 & 1 & 38.1 & 19 & 58.2 \\
$h_{0}$ & & & & 32.8 & 11.1 & 88.6 & 46.1 & 137.9 \\
$s_{0}$ & & & & & 12.8 & 19.2 & 6.9 & 34.2 \\
$m$ & & sym & & & & $\mathbf{2 7 9 . 4}$ & 139 & 427.7 \\
$a$ & & & & & & & 74.8 & 212.2 \\
$\xi$ & & & & & & & & $\mathbf{3 8 7 . 4}$
\end{tabular}

Table 4: The normalized sensitivity matrix for loading case $\mathrm{D}$ also shows that $\phi_{1}$ is not sensitive to parameters $h_{0}, n, C$ and $s_{0}$ (italics) but very sensitive to parameters $m, \xi$ and $\tilde{s}$ (bold).

element study shows that the cost function does not depend significantly on the stress ratio, this configuration is as well suited as any other for the purpose of material parameter identification. However, it has two distinct advantages: it ensures heterogeneity in the strain field $(65)$ and can be used directly in a universal test machine without the need for special fixtures (66). The solder joint is chosen to be a square of side $3 \mathrm{~mm}$ in the plane. This choice ensures that the joint is representative of those in real applications, and the entire field of view is contained well within the commonly employed 4:3 aspect ratio image sensor even at large displacement. Thus, the spatial resolution of the kinematic variables, which plays an important role in the identification process (53), is not compromised at any time during the loading. However, imaging such a small region of interest calls for a high-magnification set-up with a long working distance. For instance, if a camera with $2000 \times 2000$ pixels is used, then image pixel size will be $3000 / 2000=1.5$ micron. If the camera has a pixel size of 3.45 micron (e.g. AVT Manta camera), then a magnification of 3.45/1.5 $=2.3$ is required, which can be achieved with a macro lens. For example, the Canon MP-E 65mm macro lens (67) can be used to achieve this magnification at a working distance of $240 \mathrm{~mm}$. However, care should be exercised to minimize out-of-plane movements due to alignment of camera with the test specimen, grip alignment issues, fixture deformation, etc. (68). 


\begin{tabular}{l|llllllll} 
& $C$ & $\tilde{s}$ & $n$ & $h_{0}$ & $s_{0}$ & $m$ & $a$ & $\xi$ \\
\hline$C$ & 1.4 & 20.1 & 6.2 & 4.1 & 1 & 21.1 & 5.9 & 24.3 \\
$\tilde{s}$ & & $\mathbf{2 6 7 . 8}$ & 83.4 & 40.5 & 4.4 & 265.3 & 71.7 & 303.7 \\
$n$ & & & 25.5 & 12.3 & 1.3 & 81.3 & 21.9 & 93.1 \\
$h_{0}$ & & & & 18.5 & 6.6 & 57.6 & 20.8 & 67.6 \\
$s_{0}$ & & & & & 6.7 & 15.4 & 3.2 & 18.6 \\
$m$ & & \multirow{2}{*}{ sym } & & & & $\mathbf{2 8 1 . 9}$ & 80.1 & 326.3 \\
$a$ & & & & & & & 28.5 & 922.9 \\
$\xi$ & & & & & & & & $\mathbf{2 3 4 . 2}$
\end{tabular}

Table 5: The normalized sensitivity matrix for simple-shear loading case $\mathrm{A}$ at a higher strain rate of $10^{-1} \mathrm{~s}^{-1}$ is similar to that at a strain rate of $2 \times 10^{-4} \mathrm{~s}^{-1}$ (Tables 3 and 4 ) but with higher sensitivity to $n$ and balanced sensitivities for $\tilde{s}, m$ and $\xi$. Material parameters $(\tilde{s}, m$ and $\xi$ ) which significantly influence $\phi_{1}$ are indicated by bold font whereas the least influential parameters $\left(C, h_{0}\right.$ and $\left.s_{0}\right)$ are indicated by italics.

\section{Numerical results and discussion}

A finite-element model of the optimized test configuration (Fig. 8) was built and a displacement controlled simulation was performed. The global shear strain was limited to 5\% to enable the use of the infinitesimal deformation VFM formulation with negligible error in this preliminary study. After a mesh convergence study, the model was discretized into 4582 elements, of which 1600 were in the solder joint, which is the region of interest. As the primary interest was to obtain the Anand model parameters, the elastic material parameters ( $E=48 \mathrm{GPa}$ and $v=0.36$ ) were assumed to be known.

The focus was to identify a loading profile which leads to well-posedness of the inverse problem, indicated through the convergence of the gradient based minimization routine to the true material parameter set independent of initial parameter values. Therefore, in this preliminary study, three different loading profiles were tested (Fig. 9), viz., monotonic shear loading, I; shear loading at two different strain rates combined with relaxation, II; and shear loading with four different applied strain-rates and relaxations, III. The applied relaxation regimes in loading profiles II and III are shown in Fig. 10. The strain field evolution over monotonically increasing loading segments were used to compute the localized strain rates for all loading profiles and their range over 


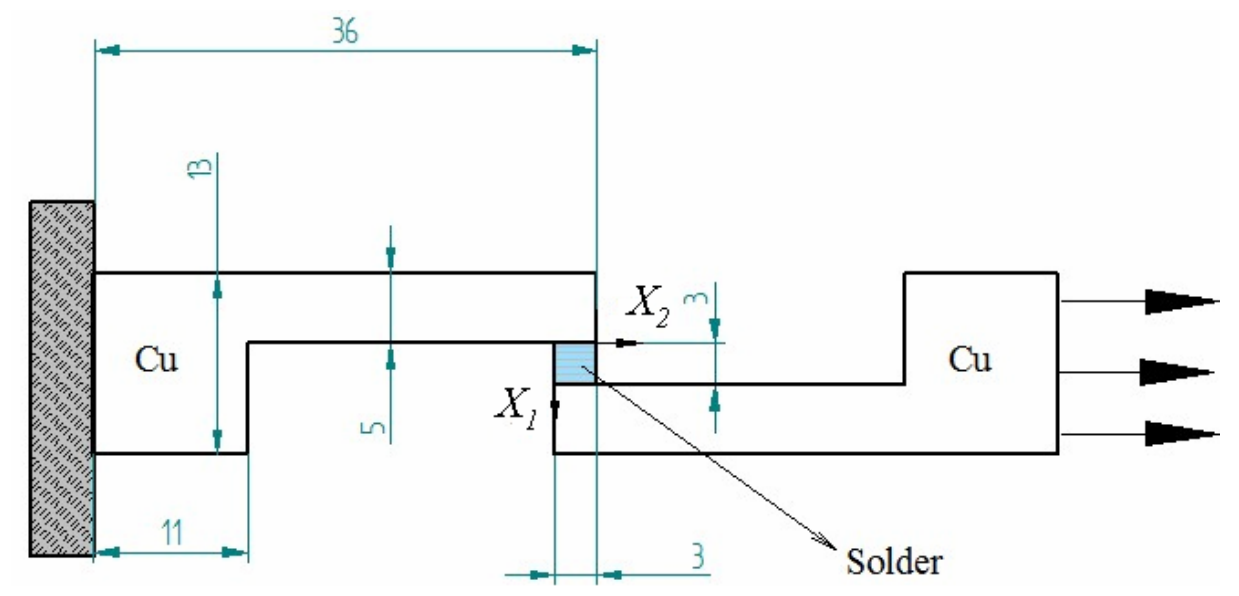

Figure 8: The modified lap shear configuration used for identification of Anand model constitutive parameters (dimensions in $\mathrm{mm}$ ).

the region of interest is shown in Fig. 11, the monotonic profile I yields strain-rates in the range $5 \times 10^{-6} \mathrm{~s}^{-1}$ to $10^{-3} \mathrm{~s}^{-1}$ even though the applied global strain-rate is held constant at $5.5 \times 10^{-4}$ $\mathrm{s}^{-1}$, primarily due to the heterogeneity of the shear strain in the region of interest; profile II yields averaged strain-rates from $10^{-5} \mathrm{~s}^{-1}$ to $2 \times 10^{-3} \mathrm{~s}^{-1}$, while the largest range from $10^{-5} \mathrm{~s}^{-1}$ to $10^{-2}$ $\mathrm{s}^{-1}$ is obtained for profile III. However, the effective strain-rate ranges relevant to VFM computations is smaller than these since the regions with smaller strain rates also have low strains and will therefore contribute little to the VFM integrals.

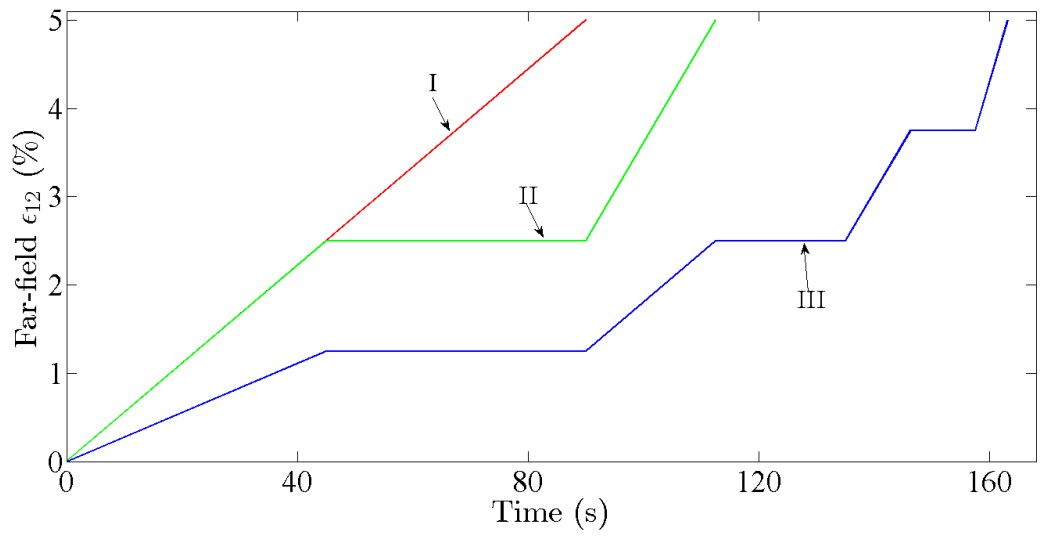

Figure 9: Applied shear strain variation: loading profile I corresponds to monotonic loading; II to two applied strain rates with relaxation and III corresponds to 4 applied strain rates with relaxation in between; all the loading profiles reach $5 \%$ global shear strain at the end. 


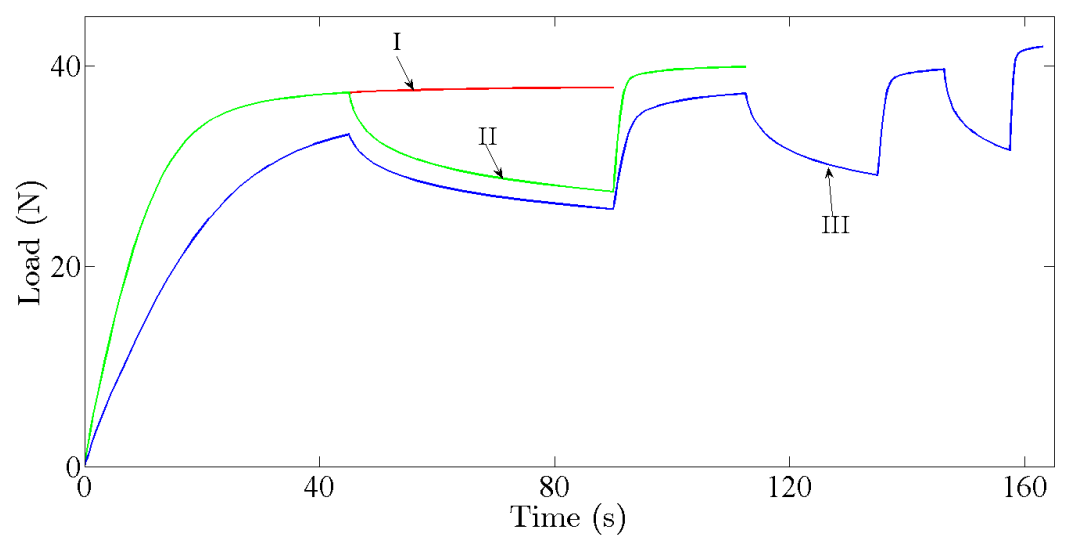

Figure 10: Load vs time plot for the three loading profiles I, II and III. Relaxation in I and II loading is clearly noticed.

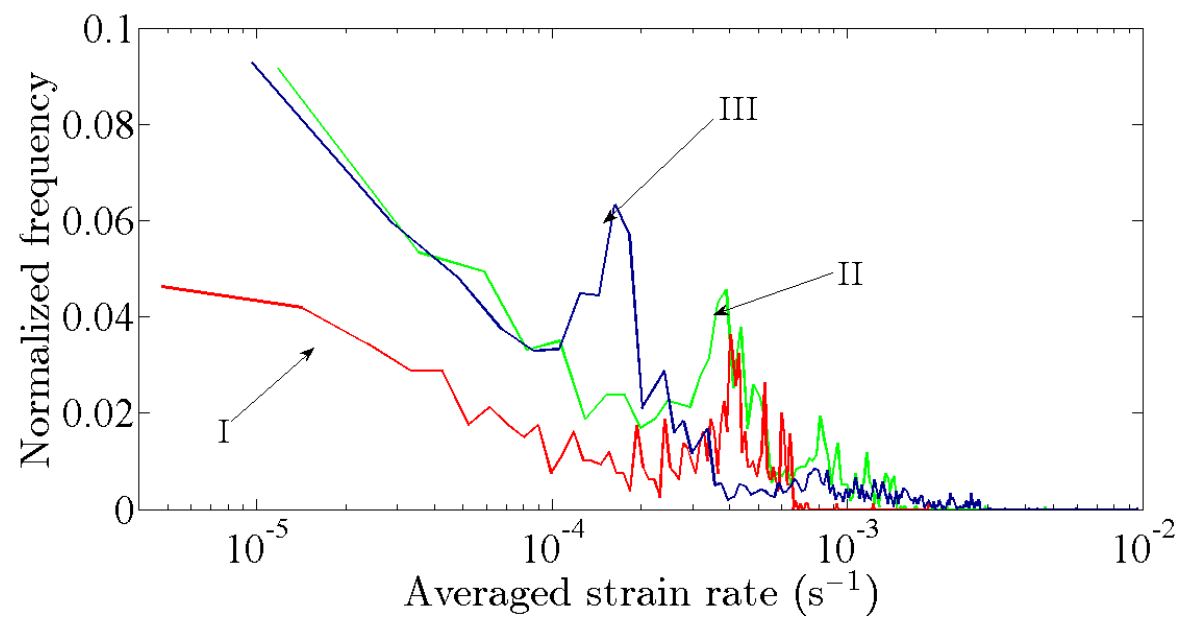

Figure 11: Averaged strain-rate sampled through the loading segments of loading profiles I, II and III are indicated through red, green and blue lines respectively. 
The components of the logarithmic strain $\mathbf{E}$ are stored at the end of each time step in the simulation. Although the present application involves infinitesimal deformation, the logarithmic strain is used so that finite deformation VFM may also be accommodated later without any change in the stress updating algorithm. A few applications of VFM-based material characterization in a finite deformation framework can be found in $(69,70 ;, 71 ; 72)$ and extension of the present work for finite deformation cases will be pursued in future work. As shown in Fig. 12, the strain components $E_{11}$, $E_{22}$ and $E_{12}$ show concentrations at the corners of the joint, which is a point of singularity that cannot be resolved by mesh refinement. In Fig. 13 , the strain fields in the interior $80 \%$ of the joint are shown and it is evident that the normal strain components are much smaller in magnitude than the shear strain in the interior of the joint. The stress fields computed using the modified stress updating algorithm are shown in Fig. 14, the shear stress $\sigma_{12}$ is the largest in magnitude and does not show large values at the corners, while the two normal stresses $\sigma_{11}$ and $\sigma_{22}$ show large values at the corners, but are small everywhere else. Since uncertainty in the computed strains and stresses are high at the corners, including them in the VFM integrals may lead to more uncertainty in the computed material parameters.

The region of interest is divided into $Z=40$ horizontal slices of equal length $X_{1}^{i+1}-X_{1}^{i}=L / Z=$ $3 / 40 \mathrm{~mm}$ and the virtual field for any $i^{\text {th }}$ slice is chosen as simple shear:

- For $X_{1} \leq X_{1}^{i}, \quad u_{1}^{* i}=u_{2}^{* i}=0$

- For $X_{1}^{i}<X_{1}<X_{1}^{i+1}, \quad u_{1}^{* i}=0$ and $u_{2}^{* i}=X_{1}$

- For $X_{1} \geq X_{1}^{i+1}, \quad u_{1}^{* i}=0$ and $u_{2}^{* i}=X_{1}^{i+1}-X_{1}^{i}$

The cost function is chosen so that the squared deviation between the external and internal virtual work over every $i^{\text {th }}$ horizontal slice of the solder and at every time step is included (61). The cost function is then normalized so that equal weights are assigned at every time step irrespective of the magnitude of load.

$$
\phi(\boldsymbol{p})=\sum_{i=1}^{Z} \sum_{j=1}^{N_{\mathrm{t}}}\left[\frac{\frac{P\left(t_{j}\right) L}{t Z}-\int_{V_{i}} \sigma_{12}(\boldsymbol{p}) d V}{\frac{P\left(t_{j}\right) L}{t Z}}\right]^{2},
$$

where $P\left(t_{j}\right)$ represents the resulting load at $j^{\text {th }}$ time step and $t$ refer to the unit thickness of the test configuration. Thus, virtual normal strains are zero, leading to zero internal virtual work from these 


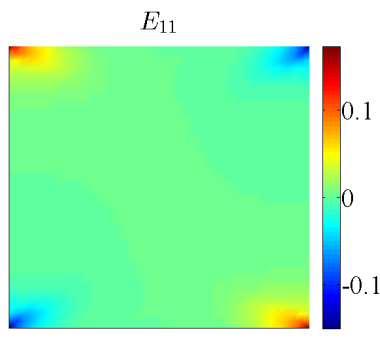

components. The only non-zero internal virtual work contribution comes from $\sigma_{12}$, which does not contain high stress gradients over the field of view.
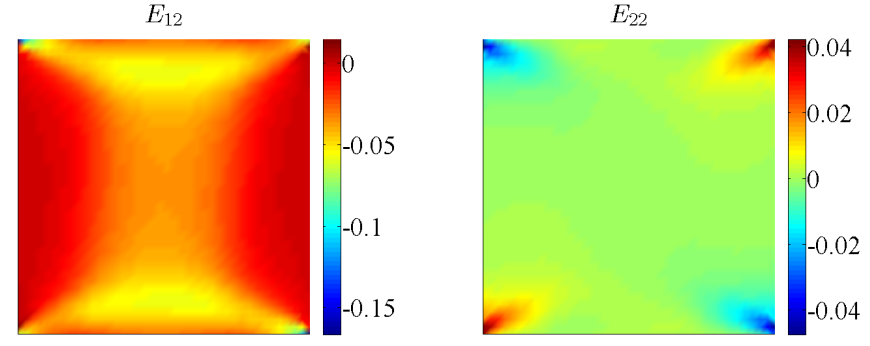

Figure 12: Logarithmic strain components at the end of the simulation for loading profile III indicate strain concentration at the corners.
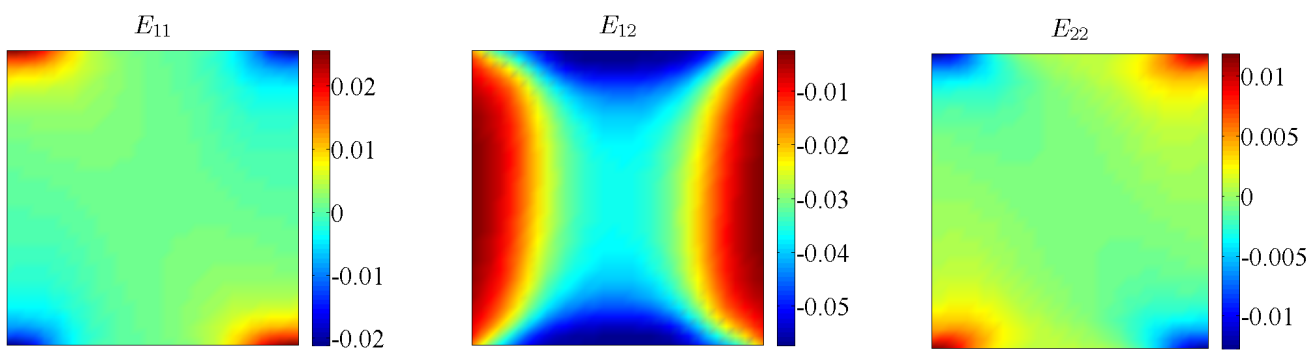

Figure 13: Logarithmic strain components at the end of simulation for loading profile III excluding the $20 \%$ region at the boundaries indicate the smaller magnitude of axial strains when compared with shear strain over most of the region of interest.

The evolution of deformation resistance $s$ shows an interesting pattern: it increases quickly from its initial value of $s_{0}$ to close to the saturation value in the first 45 seconds of deformation; and the change in $s$ through the rest of the deformation is very small, except for small jumps seen after the relaxation period. The evolution of $s$ in the central and free edge regions (points P and Q) are distinctly different (Fig. 15) as the evolution of equivalent plastic strain rates $\dot{\tilde{\varepsilon}}^{p}$ are different for the same applied global strain rate.

The cost function $\phi$ (Eqn. 11) is minimized for all the three loading profiles, I, II and III using the stresses computed from the kinematic fields and a suitable guess for the set of material parameters. As discussed in Section 2, at the outset, $\xi$ is set to be equal to 7, which leaves 7 material parameters to be obtained by the optimization procedure. Since $\phi$ is non-quadratic, the influence of 

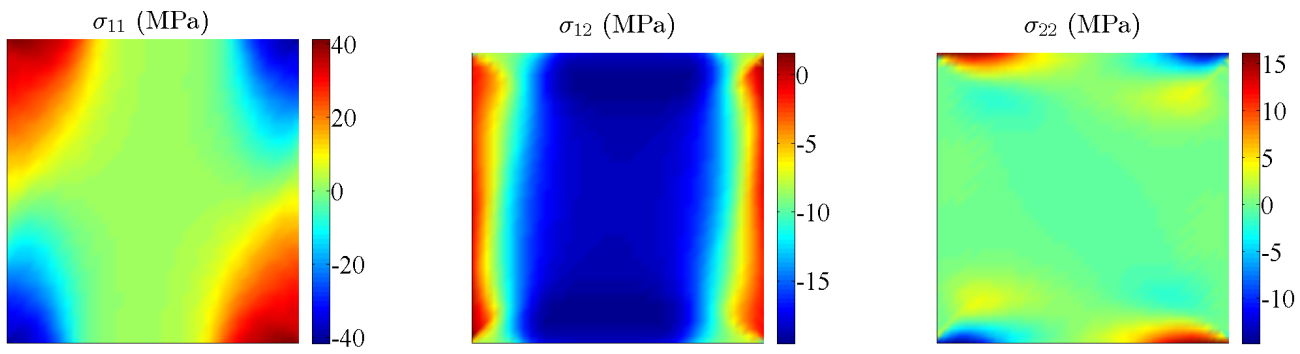

Figure 14: Cauchy stress components at the end of simulation for loading profile III indicate the prominence of shear and bending, as expected. The normal stresses are concentrated at the corners while the in-plane shear stress is nearly uniform in the central region.
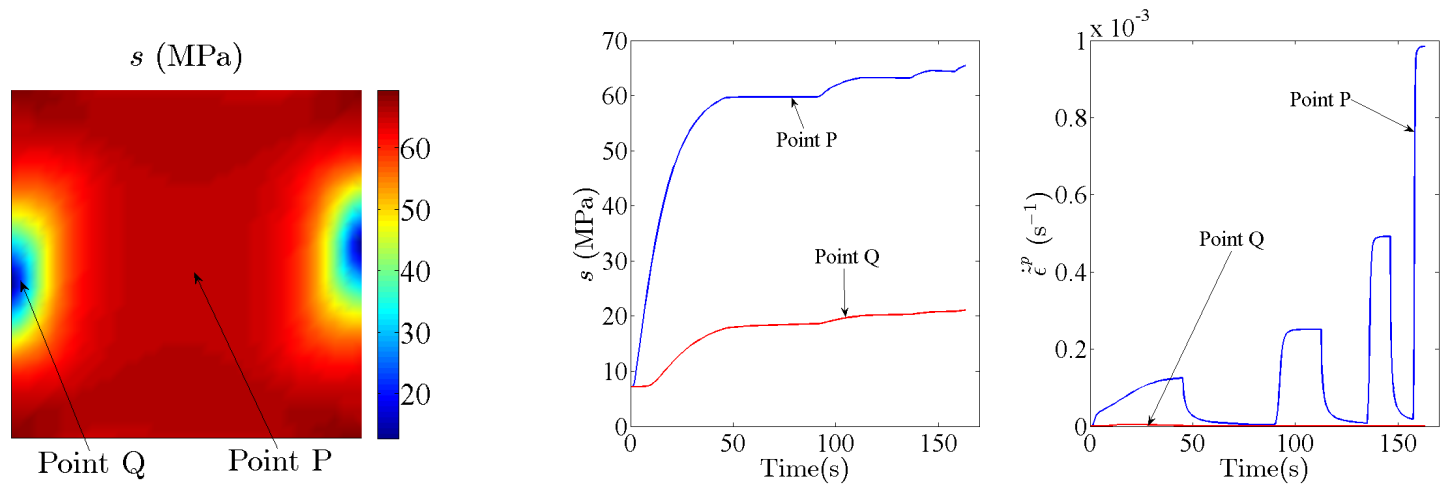

Figure 15: Deformation resistance $s$ (top) is heterogeneous. $s$ increases much more rapidly at $\mathrm{P}$ compared to $\mathrm{Q}$ due to the larger magnitudes of $\dot{\tilde{\varepsilon}}^{\mathrm{p}}$ at $\mathrm{P}$ than at $\mathrm{Q}$, where all strain components are very small (Fig. 12). 
the initial guess on the solution must be studied. This is done by using a set of 12 different initial guesses obtained via Latin Hypercube sampling ${ }^{2}(73)$ of the 7-dimensional parameter space.

As done in Section 3, the optimization is first attempted using the Matlab built-in function fminsearch. However, it is not straightforward to handle upper and lower bounds on this function. Therefore, a gradient-based method, fmincon, with the interior point algorithm is used to minimize $\phi$. The upper and lower bounds for the material parameters are chosen to be approximately $\pm 50 \%$ of their reference values and are listed in Table 6. The material parameters obtained from the optimization routine for the three loading regimes I, II and III and for all the 12 initial guesses are shown in Fig. 16. Several interesting trends can be seen in these plots. First, although the cost function for the true material set for any loading profile should be zero in principle, the computed values for each of the three profiles are not, as shown in the last sub-plot of Fig. 16. One of the reasons for this discrepancy is the way the virtual work integrals are computed. The stresses and strains are assumed to be piecewise-constant within each element, a simplification that can be expected to yield errors in high-gradient regions. Due to this error in computing the virtual work integrals, it is also seen that some of the initial guesses (e.g., the $2^{\text {nd }}, 5^{\text {th }}, 6^{\text {th }}, 7^{\text {th }}, 9^{\text {th }}, 11^{\text {th }}$ and $12^{\text {th }}$ of loading profile II) converge to cost function values that are smaller than that of the true material parameter set. In addition to the assumption of piece-wise constant strains and stresses, two other sources of error are the stress computation routine and the use of infinitesimal PVW instead of the finite deformation version. It is also noted that the cost function for the true material parameter set is seen to be non-zero even for the one-element model; since the strains are actually uniform over the entire element, the piecewise-constant assumption does not contribute to this error. Even though the converged $\phi$ values for these cases are lower than the corresponding value for the true material set, the global minimum is not attained as the parameters have converged to values different from the true parameter set. There appears to be a multitude of parameter sets whose $\phi$ values are lower than that corresponding to true parameter set. All the computed material parameters, those $(\tilde{s}, m$ and $a$ ) that were identified by the one-element study as having a strong influence on $\phi$ as well as

\footnotetext{
${ }^{2}$ Latin hypercube sampling is a technique of generating random sample sets in a higher dimensional parameter space. The randomness should obey the following restriction: if $N$ sample sets are to be generated in $M$-dimensional parameter space, then the range of each parameter is divided into $N$ equally spaced intervals and the $N$ samples are then chosen so that every interval is represented by a sample and is non-repeating among different samples in the particular parameter space. For instance, in a 2-dimensional space, if equally spaced intervals are represented by columns and the sample sets by rows, then a sample is present in every column and row.
} 


\footnotetext{
${ }^{3}$ The bottom and top horizontal lines of the box plot correspond to $25^{\text {th }}$ and $75^{\text {th }}$ percentile data respectively and the red line corresponds to the median of the dataset, while the outliers are represented by plus marks.
} 


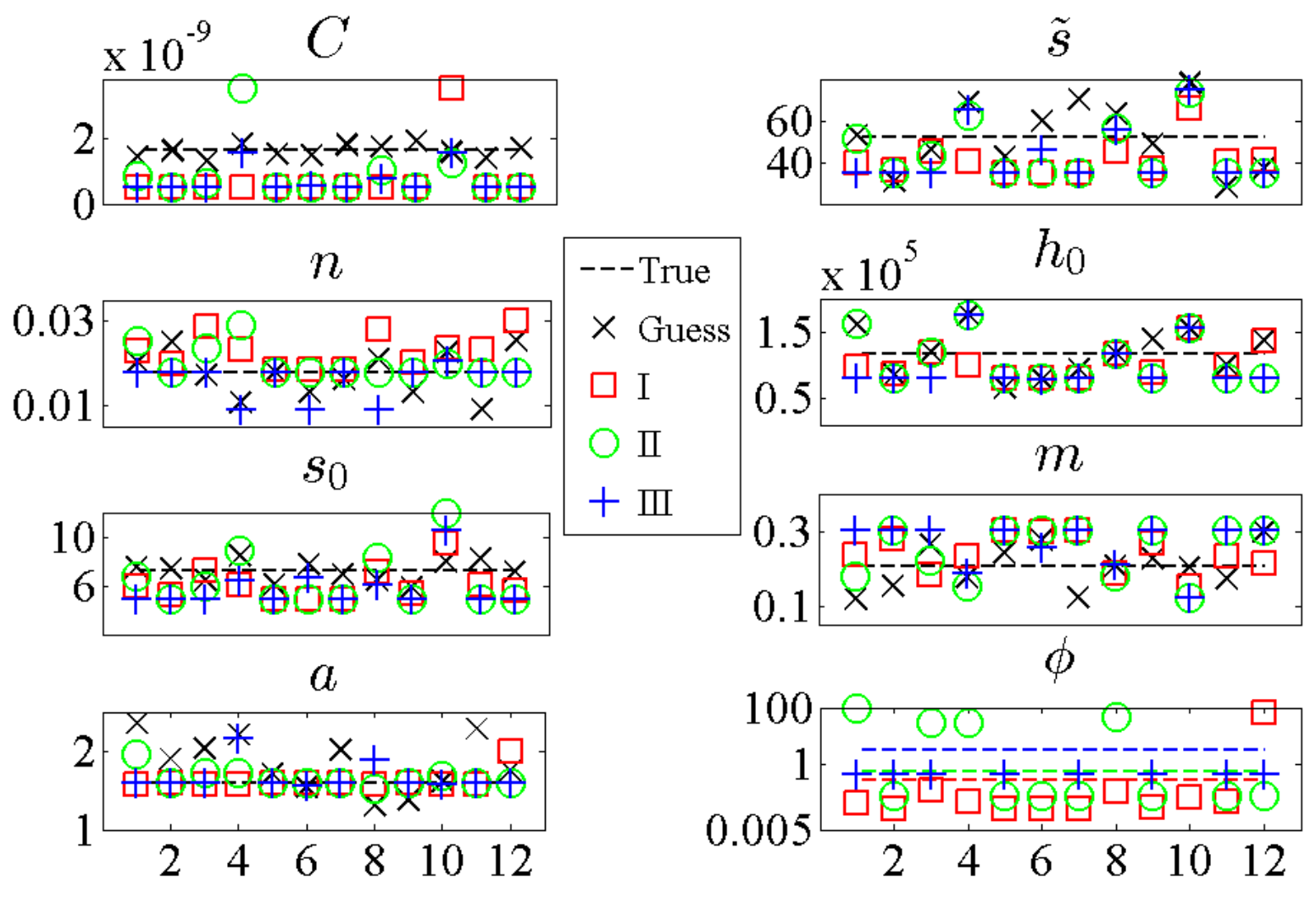

Figure 16: The same initial guess is provided to the optimizer using kinematic data from I, II and III loading profiles and non-uniqueness is observed in all cases. The cost function value at the end of convergence is also shown in the last sub-plot with the dotted lines referring to the cost function value corresponding to the true parameter set. 


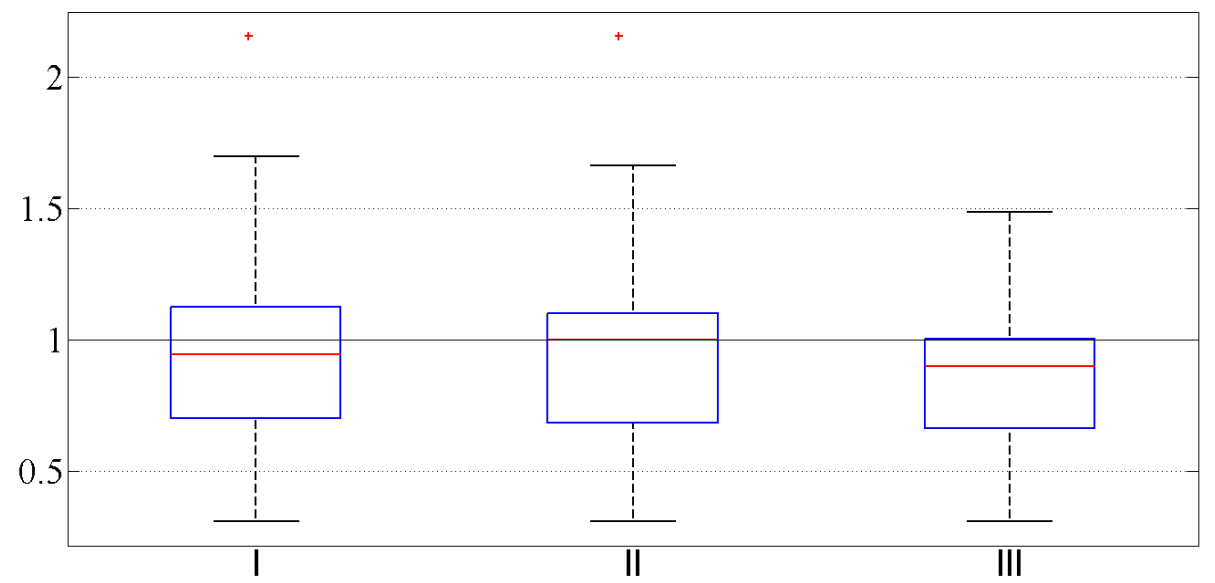

Figure 17: The box plots indicates that the variability in the estimated material parameters is significant for all the loading profiles.
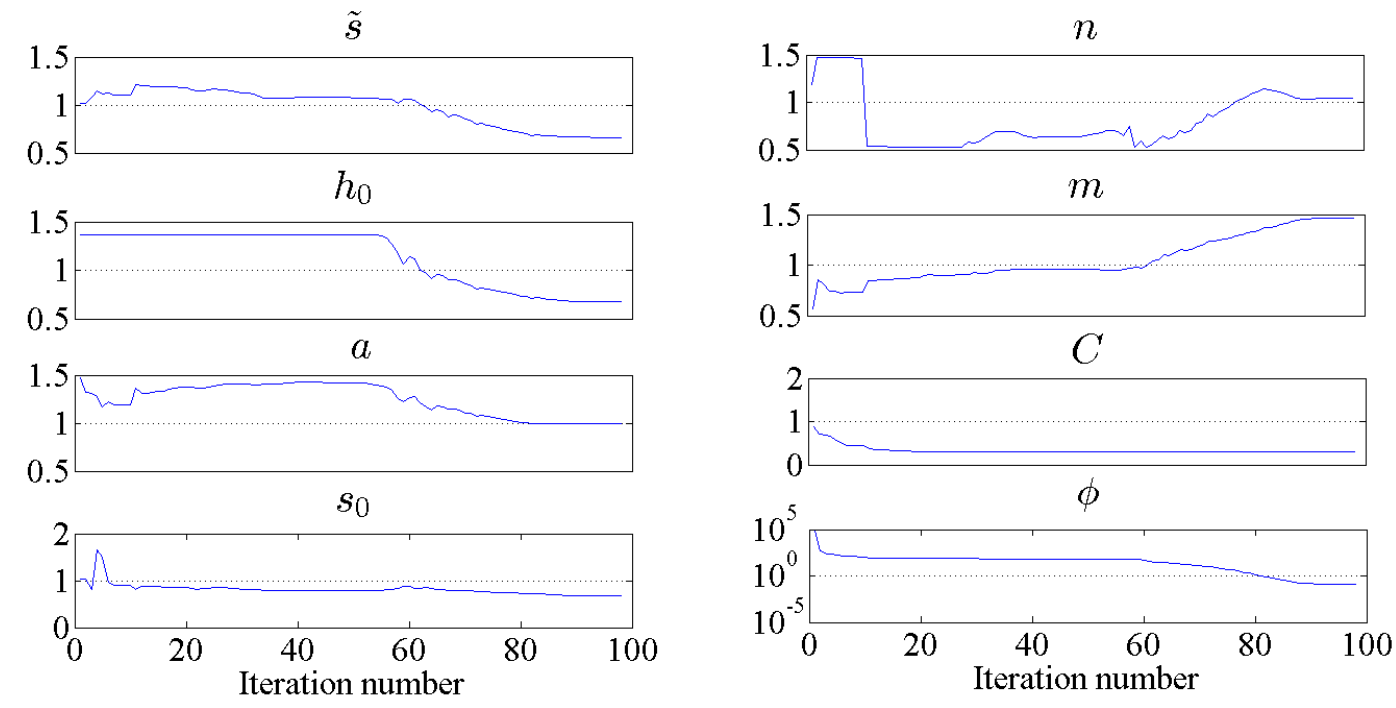

Figure 18: Evolution of the material parameters during the iterations of the gradient based minimization (all plots have been normalized with respect to the true values of respective parameters). 

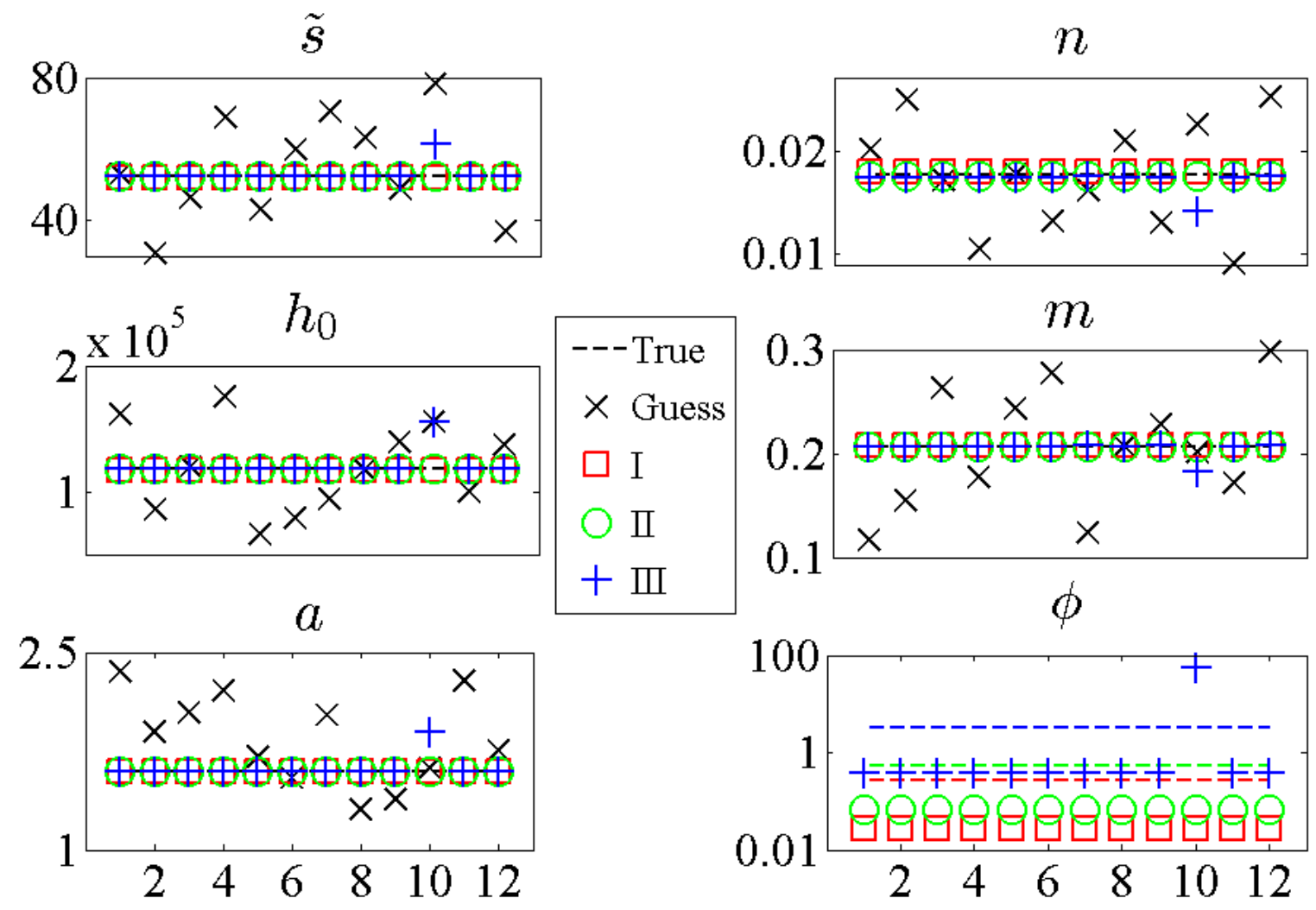

Figure 19: The same initial guess is provided to I, II and III loading profiles while $C$ and $s_{0}$ are kept fixed at their true values. Irrespective of the loading profile and the initial guess, the cost function converges to the global minimum.

In order to study the effect of the choice of cost function on the inverse technique, the cost function formulated in Section 3(Eqn. 9) with the virtual field being chosen as simple shear over the entire domain is used.

$$
\begin{aligned}
& u_{1}^{*}=0 ; u_{2}^{*}=X_{1} ; \\
& \Rightarrow \varepsilon_{11}^{*}=0 ; \quad \varepsilon_{22}^{*}=0 ; \quad \varepsilon_{12}^{*}=0.5 ;
\end{aligned}
$$

For this cost function $\phi_{1}$, the influence of the loading profile becomes much stronger when the parameters $C$ and $s_{0}$ are fixed at their true values (Fig. 21). Profile III leads to excellent identification of the parameters for all 12 initial guesses, followed by loading profile II, which leads to correct identification for 10 of the 12 initial guesses. However, profile I performs quite poorly even with $C$ 


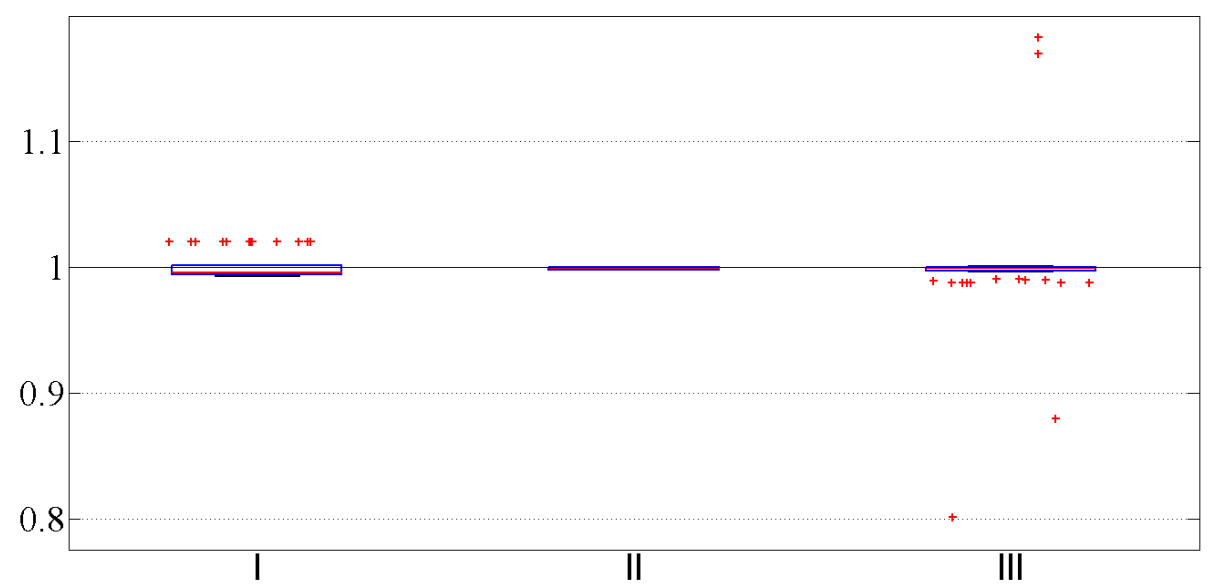

Figure 20: The box plots indicate that the converged parameters sets are very close to the true set for all loading profiles when $C$ and $s_{0}$ are fixed at their true values. The outliers of profile I correspond to parameter $n$, which is least influential among the remaining 5 parameters, while the outliers of profile III correspond to premature convergence of the $10^{\text {th }}$ initial guess as well as corresponding to parameter $n$.

and $s_{0}$ fixed at their true values throughout the optimization process, yielding the correct material parameter set for none of the initial guesses considered. Recalling that profile III has the most relaxation steps and I has none, the trend of Figs. 21 and 22 may be extrapolated to suggest that more discriminating full-field data for material parameter identification may be obtained from tests that include more stress jumps, cyclic loads, variable strain rates, etc.

Entrapment of the objective function in local minima is a major issue in inverse problems dealing with inelastic constitutive models $(59 ; 60 ; 74)$. Even though the optimizer converges and yields a material parameter set, the predictive capability of a model using such a material parameter set is questionable, as demonstrated for hyperelastic materials by Ogden et al. (75). It is worth mentioning that Andrade-Campos et al. (59) also obtained Anand visco-plastic constitutive model parameters of an $\mathrm{Al}$ alloy through a conventional inverse technique based on tension and shear experiments conducted at different temperatures and reported the occurrence of numerous local minima in their gradient-based optimization process, which prompted them to use evolutionary techniques that would enable them to reach the global minimum. The use of such global optimization techniques in the present scheme will be explored in future work.

Several works in the literature have previously obtained Anand model parameters for solders 


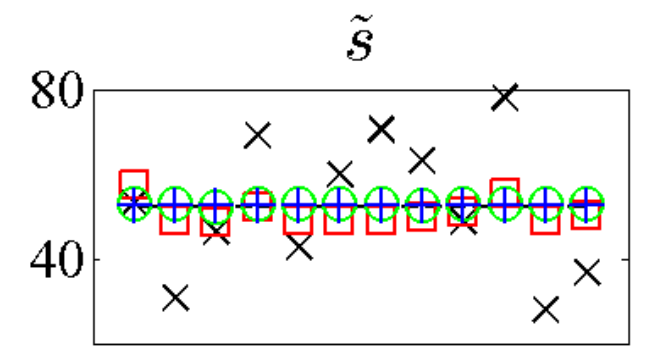

$n$
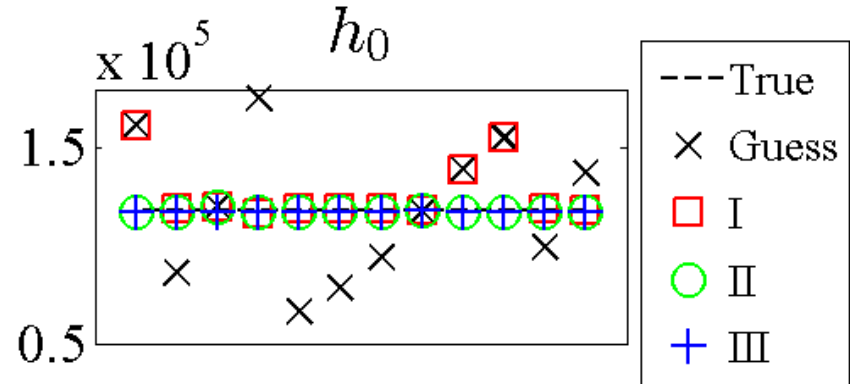

0.01
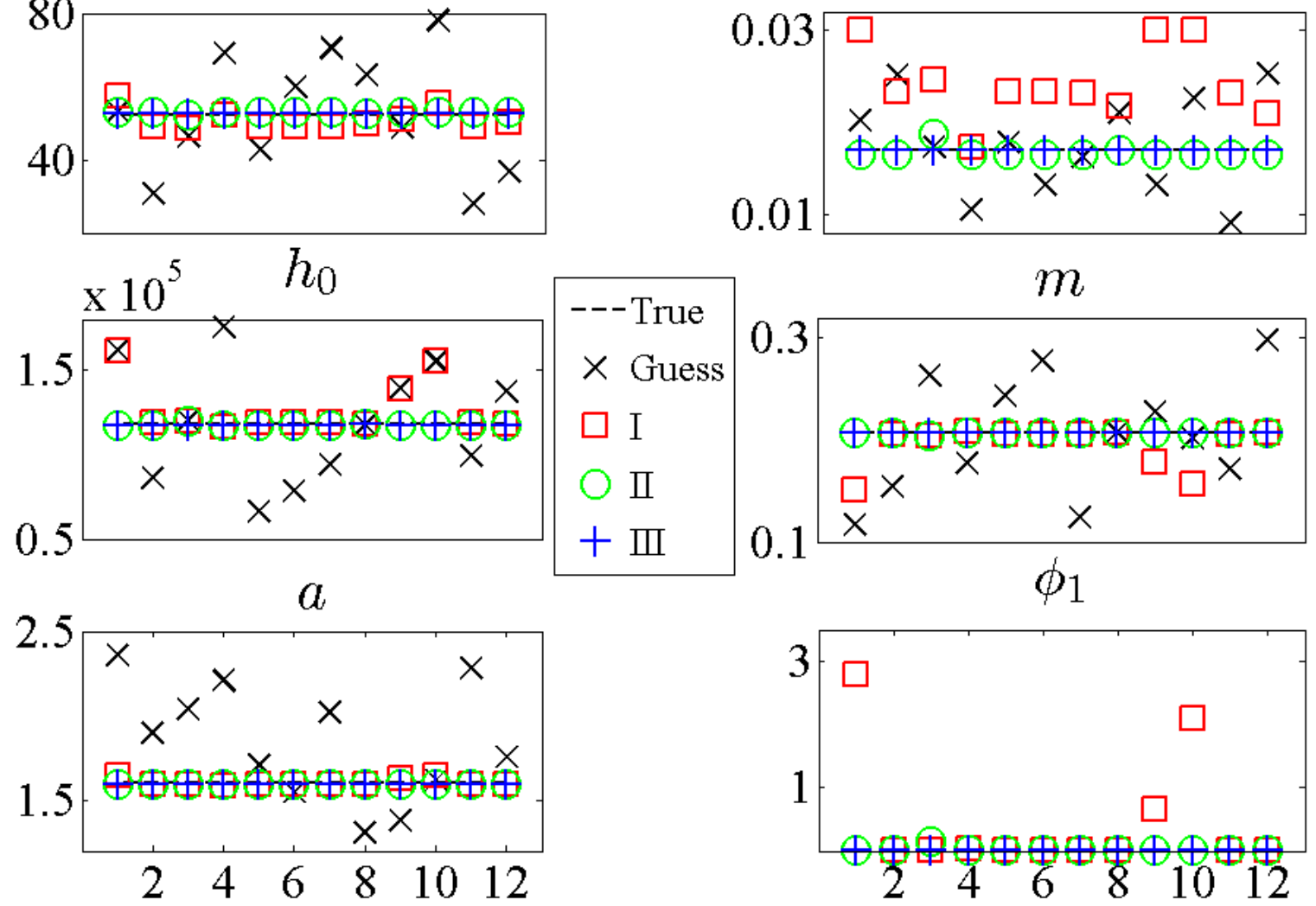

Figure 21: The same initial guess is provided to I, II and III loading profiles while $C$ and $s_{0}$ are kept fixed at their true values, but using cost function $\phi_{1}$ instead of $\phi$ (see Fig. 19). The converged material parameters for all the cases indicates that loading profile III converges to the true set for all 12 initial guess sets while II converges in 10 out of 12 guess sets; while non-uniqueness is observed for loading profile I. 


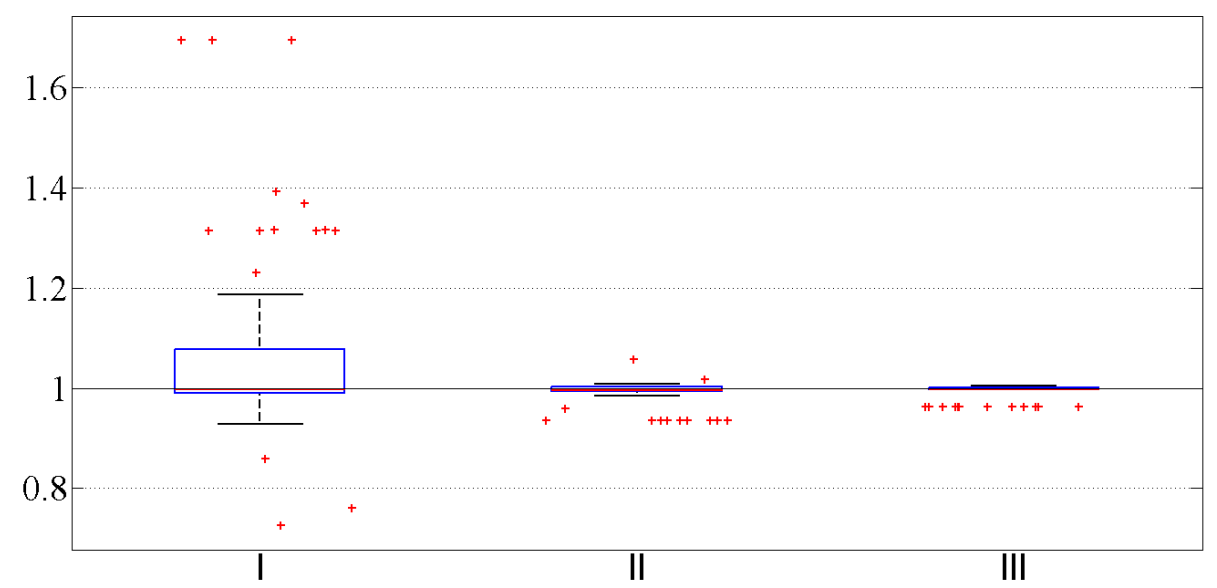

Figure 22: The box plots indicate that the variability in the estimated material parameters progressively decreases for loading profiles I, II and III, when $C$ and $s_{0}$ are fixed at their true values and $\phi_{1}$ is minimized. Loading profile III performs the best with all 12 initial guesses converging to the true material parameters set.

and thus, it is essential to place the work in context. For example, researchers $(25 ; 76 ; 77 ; 78)$ have obtained Anand material parameters for SAC 305, a popular lead-free solder alloy, using non-linear least squares fitting of uniaxial monotonic or creep test data. However, a rather large range of material parameters are reported in these studies for nominally the same material. In Fig. 23 stress-strain curves under uniaxial tension at an applied strain-rate of $0.001 \mathrm{~s}^{-1}$ at $25^{\circ} \mathrm{C}$ are plotted using the Anand model parameters from four studies (in all curves, a Young's modulus of $45 \mathrm{GPa}$ as reported by Motalab et al. (78) and a Poisson's ratio of 0.35 are used). Evidently, different responses are obtained from the different studies; in fact, the Motalab et al. (78) study yields two curves, one with parameters obtained from monotonic uniaxial test data and the other from creep test data. The reasons for this large discrepancy are not well understood, although it is quite plausible that differences in microstructure in the test specimens is a prominent factor. Additionally, since the constitutive equation is highly nonlinear, the issue of uniqueness discussed in the present study may also be expected to be an important contributor to this discrepancy. Each of these cited studies appears to arrive at an optimal value of the material parameters, but due to the lack of uniqueness, each set of parameters produces a significantly different macroscopic response. This problem may be expected to become more pronounced if responses to multi-axial stress states 
are sought.

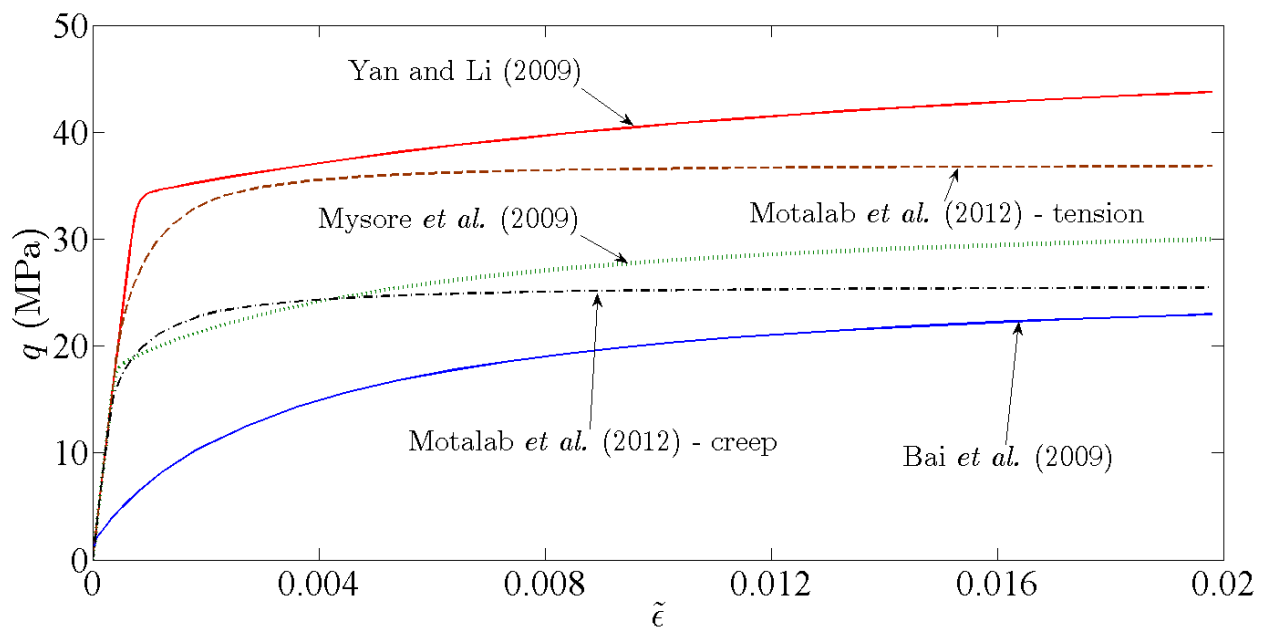

Figure 23: Equivalent stress-strain responses computed from Anand model parameter sets of SAC 305 alloy available in literature show significant variation. The curves are obtained when global strain rate is $0.001 \mathrm{~s}^{-1}$ and the temperature is $25^{\circ} \mathrm{C}$.

Since materials scientists are often interested in the role of microstructure on macroscopic properties, the present study offers an important word of caution: before one can attempt to study structure-property relationships, especially in the case of complicated constitutive models, one must resolve the aforementioned uniqueness issues. For if these are not dealt with properly, the chosen numerical scheme may lead to misidentification of material properties, thereby leading to an incorrect understanding of the role of microstructure in determining constitutive properties. Specifically, straightforward nonlinear least squares fitting of limited test data such as those from uniaxial or shear experiments may be insufficient. Test data of the type explored in the current study are more suitable for material property estimation; the more heterogeneous the training data sets are with respect to strains, strain rates and temperatures, the more robust they will be with respect to material property identification.

\section{Concluding remarks}

In the present work, the issue of identifiability of the Anand visco-plastic model constitutive parameters using VFM and synthetic full-field kinematic data is investigated. VFM has been used for the first time to characterize an inelastic material model with more than 4 material parame- 
ters. A modified lap-shear specimen is designed and three different loading profiles are used in a finite-element model of this specimen to generate synthetic full-field kinematic data for the inverse computations. The following conclusions are drawn from this work:

- A preliminary single-element study performed over a range of normal to shear stress shows that the VFM cost-function is not sensitive to the loading direction.

- The single-element study also shows that the cost function is sensitive to $\tilde{s}, m, a$ and $\xi$, but not so sensitive to parameters $h_{0}, s_{0}, C$ or $n$.

- Due to the form of the Anand model constitutive equations, one can obtain the four parameters $\tilde{s}, h_{0}, h_{0}$ and $\xi$ to only within a multiplicative constant.

- The formulation of the cost function to be minimized plays an important role in the inverse technique.

- VFM computations with 12 different initial guesses show that both the loading profile and initial guess have a significant impact on the obtained material parameter set.

- The two parameters $C$ and $s_{0}$ have the least impact on the cost function and the gradient based minimization technique is not able to drive them towards their true values. This is supported by the observation that estimates improve dramatically for all loading profiles once these values are fixed at their true values.

- In order to obtain material parameters from a gradient based optimization technique, the cost function should be formulated such that it is almost equally sensitive to all the parameters. As the functional form of a constitutive model directly affects this issue, care needs to be exercised during the development of the constitutive model.

- More complicated loading profiles with stress jumps, multiple strain rates, cyclic loading, etc. are likely to reduce non-uniqueness in the computed parameters.

- The gradient-based optimization scheme employed may be substituted by a global optimization scheme to avoid the issue of entrapment in local minima altogether, albeit at a greater computational expense. 


\section{Acknowledgements}

SNG and SJS thank IIT Madras and the Ministry of Human Resource Development, Government of India for funding this research work. They also thank Dr. Ireneusz Lapczyk of Dassault Systèms Simulia Corp. for help with the stress integration routine in Abaqus and SNG also thanks the IIT Madras-Paristech Ph.D. mobility programme for funding a 6-month stay at Arts et Métiers ParisTech, Châlons en Champagne, France.

\section{Appendix A - Stress updating algorithm for Anand visco-plastic model (63) applicable to plane stress}

The Anand model was developed to describe hot working of initially isotropic materials with isotropic hardening using the state variables $[\boldsymbol{\sigma}, s, \theta]$, where $\boldsymbol{\sigma}$ is the Cauchy stress, $s$ is a scalar internal variable representing the isotropic resistance offered by the material to plastic deformation and $\theta$ is the temperature. The evolution equation for the Cauchy stress is given through

$$
\boldsymbol{\sigma}^{\nabla}=\mathscr{L}\left[\mathbf{D}-\mathbf{D}^{\mathrm{p}}\right]
$$

where $\boldsymbol{\sigma}^{\nabla}$ is the Jaumann derivative of the Cauchy stress $\boldsymbol{\sigma}, \mathscr{L}$ is the elasticity tensor, $\mathbf{D}$ is the rate of deformation tensor and $\mathbf{D}^{p}$ is the plastic part of $\mathbf{D}$. The flow rule is given by

$$
\mathbf{D}^{\mathrm{p}}=\dot{\tilde{\varepsilon}}^{\mathrm{p}}\left[\frac{3}{2} \frac{\boldsymbol{\sigma}^{\prime}}{q}\right]
$$

where $\boldsymbol{\sigma}^{\prime}$ is the deviatoric part of the Cauchy stress, $\dot{\tilde{\varepsilon}}^{\mathrm{p}}=\mathrm{f}(q, \theta, s)>0$ is the equivalent plastic strain rate, i.e., a function of von-Mises stress $q$, internal variable $s$ and temperature $\theta$. The evolution of $s$ is given by

$$
\dot{s}=g(q, \theta, s)
$$

During finite deformations, material frame-indifference restricts the form of constitutive model so that no stress increment is measured by a co-rotational observer for pure rotation. Since the basis also spins along with the material, the rotation tensor, $\mathbf{Q}(\zeta)$ used to ensure material frame- 

sit

indifference is to be found through the solution of the initial value problem (79)

$$
\dot{\mathbf{Q}}(\zeta)=\mathbf{W}(\zeta) \mathbf{Q}(\zeta) ; \quad t \leq \zeta \leq \tau
$$

with the initial conditions $\mathbf{Q}(t)=\mathbf{I}$ and $\mathbf{W}(\zeta)$ represents a spin tensor at time $\zeta$. Using $\mathbf{Q}(\zeta)$, Lush et al. (63) define the bar transformation wherein the field values obtained in the material frame of reference are transformed back to the fixed reference frame through

$$
\overline{\boldsymbol{\sigma}}(\zeta)=\mathbf{Q}^{\mathrm{T}}(\zeta) \boldsymbol{\sigma}(\zeta) \mathbf{Q}(\zeta)
$$

From Eqns. (16) and (17),

$$
\dot{\overline{\boldsymbol{\sigma}}}(\zeta)=\mathbf{Q}^{\mathrm{T}}(\zeta) \boldsymbol{\sigma}^{\nabla}(\zeta) \mathbf{Q}(\zeta)
$$

It is assumed that the field values $\left(\boldsymbol{\sigma}^{k}, s^{k}\right)$ at time $t^{k}$ are known and the objective is to determine the field values at time $t^{k+1}$, i.e. $\left(\boldsymbol{\sigma}^{k+1}, s^{k+1}\right)$. Using Eqns. 13 , 17$)$,

$$
\begin{aligned}
\boldsymbol{\sigma}^{k+1} & =\mathbf{Q}^{k+1}\left[\boldsymbol{\sigma}^{k}+\int_{t^{k}}^{t^{k+1}} \mathscr{L}\left[\overline{\mathbf{D}}-\frac{3}{2} \dot{\tilde{\varepsilon}}^{\mathrm{p}} \frac{\overline{\boldsymbol{\sigma}}^{\prime}}{q}\right] d t\right]\left(\mathbf{Q}^{k+1}\right)^{T} \\
s^{k+1} & =s^{k}+\int_{t^{k}}^{t^{k+1}} \dot{s} d t
\end{aligned}
$$

Here, $\mathbf{Q}^{k+1}$ can be chosen to be the incremental rotation $(80)$, i.e. the rotation of the configuration at time $t^{k+1}$ relative to that at time $t^{k}$, obtained from the polar decomposition of relative deformation gradient, $\mathbf{F}_{\mathbf{r}}{ }^{k+1}=\mathbf{F}^{k+1}\left(\mathbf{F}^{k}\right)^{-1}$. Using Euler's backward integration scheme, Eqns. 19.20 can be written as

$$
\begin{aligned}
\boldsymbol{\sigma}^{k+1} & =\boldsymbol{\sigma}_{*}^{k+1}-6 \mu \delta t f\left(q^{k+1}, s^{k+1}\right) \sqrt{\frac{3}{2}} \frac{\left(\boldsymbol{\sigma}_{*}^{\prime}\right)^{k+1}}{\left(q_{*}\right)^{k+1}} \\
s^{k+1} & =s^{k}+\delta \operatorname{tg}\left(q^{k+1}, s^{k+1}\right)
\end{aligned}
$$

where $\boldsymbol{\sigma}_{*}^{k+1}=\overline{\boldsymbol{\sigma}}^{k}+\mathscr{L}[\boldsymbol{\delta} \mathbf{E}]$ is the trial Cauchy stress, with $\tilde{\boldsymbol{\sigma}}^{k}=\mathbf{Q}^{k+1} \boldsymbol{\sigma}^{k}\left(\mathbf{Q}^{k+1}\right)^{T}$ representing the co-rotational Cauchy stress at time $t^{k}, q_{*}^{k+1}$ denotes the trial equivalent stress, while $\delta \mathbf{E}=$ $\mathbf{Q}^{k+1}\left[\int_{t^{k}}^{t^{k+1}} \overline{\mathbf{D}} d t\right]\left(\mathbf{Q}^{k+1}\right)^{T}$. Taking the deviatoric part of Eqn. 21 and using the fact that the 
incremental plastic strain direction is along the deviatoric stress tensor, i.e., perpendicular to the yield surface, one obtains

$$
q^{k+1}=q_{*}^{k+1}-3 \mu \delta t\left(\dot{\tilde{\varepsilon}}^{\mathrm{p}}\right)^{k+1}
$$

Thus, the problem reduces to solving for $s^{k+1}$ and $q^{k+1}$ from the pair of scalar equations 22,23 . The radial-return factor is then obtained as

$$
\eta^{k+1}=\frac{q^{k+1}}{q_{*}^{k+1}}
$$

and the Cauchy stress is updated through

$$
\boldsymbol{\sigma}^{k+1}=\eta^{k+1}\left(\boldsymbol{\sigma}_{*}^{\prime}\right)^{k+1}+\frac{1}{3} \operatorname{tr}\left(\boldsymbol{\sigma}_{*}^{k+1}\right) \mathbf{I}
$$

This algorithm (63) is applicable only for plane-strain and 3D elements; the corresponding modification for its applicability to plane stress cases is done through nested iterations at the integration point level (64). Here, the out-of-plane elastic strain is updated at the integration point level in every iteration until the chosen plane stress tolerance, $\beta=5 \times 10^{-3} \mathrm{MPa}$ is achieved:

$$
\left(E_{33}^{\mathrm{e}}\right)^{k+1}=\frac{-v}{1-v}\left[\left(E_{11}^{\mathrm{e}}\right)^{k+1}+\left(E_{22}^{\mathrm{e}}\right)^{k+1}\right]-\sigma_{33}^{k+1} /\left[\frac{E(1-v)}{(1+v)(1-2 v)}\right]
$$

The pseudo-code of the stress-updating algorithm for the Anand model modified for plane stress situations is shown in Algorithm 1. 
input : Logarithmic strain at $k$ and $k+1$ increments $\mathbf{E}^{k}, \mathbf{E}^{k+1}$; Cauchy stress $\boldsymbol{\sigma}^{k}$, deformation gradient $\mathbf{F}^{k+1}$, material parameters $\boldsymbol{p}$, plane stress tolerance $\beta$, no. of elements $N_{\mathrm{e}}$, trial out-of-plane elastic strain $\left(E_{*}\right)_{33}^{\mathrm{e}}=0$

output: kinetic field at increment $k+1$

Trial incremental stress, $\delta \boldsymbol{\sigma}_{*}^{k+1}=\lambda \operatorname{tr}\left(\mathbf{E}^{k+1}-\mathbf{E}^{k}\right) \mathbf{I}+2 \mu\left(\mathbf{E}^{k+1}-\mathbf{E}^{k}\right)$

for $i \leftarrow 1$ to $N_{\mathrm{e}}$ do

while $\sigma_{33}^{k+1} \geq \beta$ do

Relative deformation gradient, $\mathbf{F}_{\mathbf{r}}{ }^{k+1}=\mathbf{F}^{k+1}\left(\mathbf{F}^{k}\right)^{-1}$;

Cauchy-Green left stretching tensor, $\mathbf{V}_{\mathbf{r}}{ }^{k+1}=\sqrt{\mathbf{F}_{\mathbf{r}}{ }^{k+1}\left(\mathbf{F}_{\mathbf{r}}{ }^{k+1}\right)^{T}}$;

Incremental rotation, $\mathbf{Q}^{k+1}=\mathbf{V}_{\mathbf{r}}{ }^{k+1}\left(\mathbf{F}_{\mathbf{r}}{ }^{k+1}\right)^{-1}$;

Co-rotational Cauchy stress, $\tilde{\boldsymbol{\sigma}}^{k}=\mathbf{Q}^{k+1} \boldsymbol{\sigma}^{k}\left(\mathbf{Q}^{k+1}\right)^{T}$;

Trial Cauchy stress, $\boldsymbol{\sigma}_{*}^{k+1}=\tilde{\boldsymbol{\sigma}}^{k}+\boldsymbol{\delta} \boldsymbol{\sigma}_{*}^{k+1}$;

Co-rotational logarithmic strain, $\tilde{\mathbf{E}}^{k+1}=\mathbf{Q}^{k+1} \mathbf{E}^{k}\left(\mathbf{Q}^{k+1}\right)^{T}+\left(\mathbf{E}^{k+1}-\mathbf{E}^{k}\right)$;

Trial deviatoric stress, $\left(\boldsymbol{\sigma}_{*}^{\prime}\right)^{k+1}=\boldsymbol{\sigma}_{*}^{k+1}-\frac{1}{3} \operatorname{tr}\left(\boldsymbol{\sigma}_{*}^{k+1}\right) \mathbf{I}$;

Trial equivalent stress, $q_{*}^{k+1}=\sqrt{\frac{3}{2} \boldsymbol{\sigma}_{*}^{k+1}: \boldsymbol{\sigma}_{*}^{k+1}}$;

Calculate $s^{k+1}$ and $q^{k+1}$ by solving

$$
\begin{aligned}
& s^{k+1}-s^{k}-\delta \operatorname{tg}\left(q^{k+1}, s^{k+1}\right)=0 \\
& q^{k+1}-q_{*}^{k+1}+3 \mu \delta t f\left(q^{k+1}, s^{k+1}\right)=0 ;
\end{aligned}
$$

Radial return factor, $\eta^{k+1}=\frac{q^{k+1}}{q_{*}^{k+1}}$;

Cauchy stress, $\boldsymbol{\sigma}^{k+1}=\eta^{k+1}\left(\boldsymbol{\sigma}_{*}\right)^{k+1}+\frac{1}{3} \operatorname{tr}\left(\boldsymbol{\sigma}_{*}\right)^{k+1} \mathbf{I}$;

Elastic strain, $\left(\tilde{\mathbf{E}}^{\mathbf{e}}\right)^{k+1}=\mathscr{L} \boldsymbol{\sigma}^{k+1}$;

Plastic strain, $\left(\tilde{\mathbf{E}}^{\mathbf{p}}\right)^{k+1}=\tilde{\mathbf{E}}^{k+1}-\left(\tilde{\mathbf{E}}^{\mathbf{e}}\right)^{k+1}$;

Elastic strain in fixed basis, $\mathbf{E}^{\mathbf{e}}=\left(\mathbf{Q}^{k+1}\right)^{T}\left(\tilde{\mathbf{E}}^{\mathbf{e}}\right)^{k+1} \mathbf{Q}^{k+1}$;

Plastic strain in fixed basis, $\mathbf{E}^{\mathbf{p}}=\left(\mathbf{Q}^{k+1}\right)^{T}\left(\tilde{\mathbf{E}}^{\mathbf{p}}\right)^{k+1} \mathbf{Q}^{k+1}$;

Elastic strain updating,

$$
\left(E_{33}^{\mathrm{e}}\right)^{k+1}=\frac{-v}{1-v}\left[\left(E_{11}^{\mathrm{e}}\right)^{k+1}+\left(E_{22}^{\mathrm{e}}\right)^{k+1}\right]-\sigma_{33}^{k+1} /\left[\frac{E(1-v)}{(1+v)(1-2 v)}\right]
$$

Plastic strain updating, $\left(E_{33}^{\mathrm{p}}\right)^{k+1}=-\left[\left(E_{11}^{\mathrm{p}}\right)^{k+1}+\left(E_{22}^{\mathrm{p}}\right)^{k+1}\right]$

\section{end}

Deformation gradient updating, $F_{33}^{k+1}=1+\left(E_{33}^{\mathrm{e}}\right)^{k+1}+\left(E_{33}^{\mathrm{p}}\right)^{k+1}$;

end $F_{13}^{k+1}=F_{23}^{k+1}=F_{31}^{k+1}=F_{32}^{k+1}=0$;

Algorithm 1: Pseudo-code of Anand visco-plastic model stress updating algorithm for plane stress, based on Lush et al. (63) 


\section{References}

[1] G. R. Johnson, W. H. Cook, A constitutive model and data for metal subjected to large strains, high strain rates and high temperatures, In Proceedings of the Seventh Symposium on Ballistics, The Hague, The Netherlands (1983) 541-547.

[2] P. Perzyna, Fundamental Problems in Viscoplasticity, edited by H. L. Dryden, Advances in Applied Mechanics, Academic Press New-York, 1966.

[3] E. Krempl, J. J. McMahon, D. Yao, Viscoplasticity based on overstress with a differential growth law for the equilibrium stress, Mechanics of Materials 5 (1) (1986) 35-48.

[4] F. J. Zerilli, R. W. Armstrong, Dislocation-mechanics-based constitutive relations for material dynamics calculations, Journal of Applied Physics 61 (5) (1987) 1816-1825.

[5] L. Lindgren, K. Domkin, S. Hansson, Dislocations, vacancies and solute diffusion in physical based plasticity model for AISI 316L, Mechanics of Materials 40 (11) (2008) 907-919.

[6] S. Nemat-Nasser, L. Ni, T. Okinaka, A constitutive model for FCC crystals with application to polycrystalline OFHC copper, Mechanics of Materials 30 (4) (1998) 325-341.

[7] S. Nemat-Nasser, T. Okinaka, N. Ni, A physically-based constitutive model for BCC crystals with application to polycrystalline tantalum, Journal of the Mechanics and Physics of Solids 46 (6) (1998) 1009-1038.

[8] S. R. Bodner, Y. Partom, Constitutive equations for elastic-viscoplastic strain-hardening materials, Journal of Applied Mechanics 42 Ser E (2) (1975) 385-389.

[9] Y. Estrin, H. Mecking, A unified phenomenological description of work hardening and creep based on one-parameter models, Acta Metallurgica 32 (1) (1984) 57-70.

[10] L. Anand, Constitutive equations for hot-working of metals, International Journal of Plasticity 1 (3) (1985) 213-231.

[11] J. L. Chaboche, A review of some plasticity and viscoplasticity constitutive theories, International Journal of Plasticity 24 (10) (2008) 1642-1693. 
[12] P. M. Naghdi, A critical review of the state of finite plasticity, Zeitschrift für angewandte Mathematik und Physik 41 (3) (1990) 315-394.

[13] A. S. Krausz, K. Krausz (Eds.), Unified Constitutive Laws of Plastic Deformation, Academic Press Inc., 1996.

[14] A. K. Miller (Ed.), Unified constitutive equations for creep and plasticity, Elsevier Applied Science, 1987.

[15] S. Nemat-Nasser, Plasticity: a treatise on finite deformation of heterogeneous inelastic materials, Cambridge University Press, 2004.

[16] S. B. Brown, K. H. Kim, L. Anand, An internal variable constitutive model for hot working of metals, International Journal of Plasticity 5 (2) (1989) 95-130.

[17] M. F. Horstemeyer, D. J. Bammann, Historical review of internal state variable theory for inelasticity, International Journal of Plasticity 26 (9) (2010) 1310-1334.

[18] H. Ma, J. Suhling, A review of mechanical properties of lead-free solders for electronic packaging, Journal of Materials Science 44 (5) (2009) 1141-1158.

[19] M. Kuna, S. Wippler, A cyclic viscoplastic and creep damage model for lead free solder alloys, Engineering Fracture Mechanics 77 (18) (2010) 3635-3647.

[20] X. Chen, G. Chen, Constitutive and damage model for $63 \mathrm{Sn} 37 \mathrm{~Pb}$ solder under uniaxial and torsional cyclic loading, International Journal of Solids and Structures 43 (11-12) (2006) $3596-3612$.

[21] L. Zhang, J. Han, Y. Guo, C. He, Anand model and FEM analysis of SnAgCuZn lead-free solder joints in wafer level chip scale packaging devices, Microelectronics Reliability 54 (1) (2014) 281-286.

[22] G. Z. Wang, Z. N. Cheng, K. Becker, J. Wilde, Applying Anand model to represent the viscoplastic deformation behavior of solder alloys, Journal of Electronic Packaging 123 (3) (2001) 247-253. 
[23] L. Zhang, S. Xue, L. Gao, G. Zeng, Z. Sheng, Y. Chen, S. Yu, Determination of Anand parameters for SnAgCuCe solder, Modelling and Simulation in Materials Science and Engineering 17 (7) (2009) 075014.

[24] X. Chen, G. Chen, M. Sakane, Prediction of stress-strain relationship with an improved Anand constitutive model for lead-free solder Sn-3.5Ag, IEEE Transactions on Components and Packaging Technologies 28 (1) (2005) 111-116.

[25] N. Bai, X. Chen, H. Gao, Simulation of uniaxial tensile properties for lead-free solders with modified Anand model, Materials and Design 30 (1) (2009) 122-128.

[26] G. Khatibi, M. Lederer, E. Byrne, A. Kotas, B. Weiss, H. Ipser, Characterization of stressstrain response of lead-free solder joints using a digital image correlation technique and finiteelement modeling, Journal of Electronic Materials 42 (2) (2013) 294-303.

[27] Z. L. Kowalewski, D. R. Hayhurst, B. F. Dyson, Mechanisms-based creep constitutive equations for an aluminum alloy, Journal of Strain Analysis for Engineering Design 29 (4) (1994) 309-316.

[28] B. Li, J. Lin, X. Yao, A novel evolutionary algorithm for determining unified creep damage constitutive equations, International Journal of Mechanical Sciences 44 (5) (2002) 987-1002.

[29] J. Cao, J. Lin, A study on formulation of objective functions for determining material models, International Journal of Mechanical Sciences 50 (2) (2008) 193-204.

[30] A. Andrade-Campos, R. De-Carvalho, R. A. F. Valente, Novel criteria for determination of material model parameters, International Journal of Mechanical Sciences 54 (1) (2012) 294305.

[31] ASTM E209 - 00, Standard practice for compression tests of metallic materials at elevated temperatures with conventional or rapid heating rates and strain rates, ASTM Internationaldoi:10.1520/E0209-00R10.

[32] I. Rohr, H. Nahme, K. Thoma, Material characterization and constitutive modelling of ductile high strength steel for a wide range of strain rates, International Journal of Impact Engineering 31 (4) (2005) 401-433. 
[33] A. Constantinescu, On the identification of elastic moduli from displacement-force boundary measurements, Inverse Problems in Engineering 1 (4) (1995) 293-313.

[34] E. Florentin, G. Lubineau, Identification of the parameters of an elastic material model using the constitutive equation gap method, Computational Mechanics 46 (4) (2010) 521-531.

[35] A. Moussawi, G. Lubineau, E. Florentin, B. Blaysat, The constitutive compatibility method for identification of material parameters based on full-field measurements, Computer Methods in Applied Mechanics and Engineering 265 (2013) 1-14.

[36] B. Blaysat, E. Florentin, G. Lubineau, A. Moussawi, A dissipation gap method for full-field measurement-based identification of elasto-plastic material parameters, International Journal for Numerical Methods in Engineering 91 (7) (2012) 685-704.

[37] D. Claire, F. Hild, S. Roux, Identification of a damage law by using full-field displacement measurements, International Journal of Damage Mechanics 16 (2) (2007) 179-197.

[38] G. Yun, S. Shang, A self-optimizing inverse analysis method for estimation of cyclic elastoplasticity model parameters, International Journal of Plasticity 27 (4) (2011) 576-595.

[39] K. T. Kavanagh, R. W. Clough, Finite element applications in the characterization of elastic solids, International Journal of Solids and Structures 7 (1) (1971) 11-23.

[40] M. Grédiac, Principle of virtual work and identification, Comptes rendus de l'Académie des Sciences série II (1) (1989) 1-5.

[41] F. Pierron, M. Grédiac, The Virtual Fields Method, Springer New-York, 2012.

[42] S. J. Subramanian, The eigenfunction virtual fields method, Conference Proceedings of the Society for Experimental Mechanics Series 3 (2014) 35-42.

[43] N. Nigamaa, S. J. Subramanian, Identification of orthotropic elastic constants using the Eigenfunction Virtual Fields Method, International Journal of Solids and Structures 51 (2) (2014) 295-304. 
[44] T. Nguyen, J. Huntley, I. Ashcroft, P. Ruiz, F. Pierron, A Fourier-series-based virtual fields method for the identification of 2-D stiffness distributions, International Journal for Numerical Methods in Engineering 98 (12) (2014) 917-936. doi :10.1002/nme.4665

[45] S. Avril, M. Bonnet, A. S. Bretelle, M. Grédiac, F. Hild, P. Ienny, F. Latourte, D. Lemosse, S. Pagano, E. Pagnacco, F. Pierron, Overview of identification methods of mechanical parameters based on full-field measurements, Experimental Mechanics 48 (2008) 381-402.

[46] M. Grédiac, F. Pierron, S. Avril, E. Toussaint, The virtual fields method for extracting constitutive parameters from full-field measurements: a review, Strain 42 (4) (2006) 233-253.

[47] L. E. Malvern, Introduction to the Mechanics of a Continuous Medium, Prentice Hall, 1977.

[48] F. Pierron, G. Vert, R. Burguete, S. Avril, R. Rotinat, M. R. Wisnom, Identification of the orthotropic elastic stiffnesses of composites with the virtual fields method: Sensitivity study and experimental validation, Strain 43 (3) (2007) 250-259.

[49] P. Wang, F. Pierron, O. T. Thomsen, Identification of material parameters of PVC foams using digital image correlation and the virtual fields method, Experimental Mechanics 53 (6) (2013) $1001-1015$.

[50] L. Robert, V. Velay, N. Decultot, S. Ramde, Identification of hardening parameters using finite element models and fullfield measurements: Some case studies, Journal of Strain Analysis for Engineering Design 47 (1) (2012) 3-17.

[51] H. Haddadi, S. Belhabib, Improving the characterization of a hardening law using digital image correlation over an enhanced heterogeneous tensile test, International Journal of Mechanical Sciences 62 (1) (2012) 47-56.

[52] M. H. H. Meuwissen, C. W. J. Oomens, F. P. T. Baaijens, R. Petterson, J. D. Janssen, Determination of the elasto-plastic properties of aluminium using a mixed numerical-experimental method, Journal of Materials Processing Technology 75 (1-3) (1998) 204-211.

[53] M. Rossi, F. Pierron, On the use of simulated experiments in designing tests for material characterization from full-field measurements, International Journal of Solids and Structures 49 (3-4) (2012) 420-435. 
[54] L. Pagnotta, Determining elastic constants of materials with interferometric techniques, Inverse Problems in Science and Engineering 14 (8) (2006) 801-818.

[55] L. Bruno, G. Felice, L. Pagnotta, A. Poggialini, G. Stigliano, Elastic characterization of orthotropic plates of any shape via static testing, International Journal of Solids and Structures 45 (3-4) (2008) 908-920.

[56] F. Mathieu, H. Leclerc, F. Hild, S. Roux, Estimation of elastoplastic parameters via weighted FEMU and Integrated-DIC, Experimental Mechanics, in Press.

[57] Y. Q. Wang, M. A. Sutton, H. A. Bruck, H. W. Schreier, Quantitative error assessment in pattern matching: Effects of intensity pattern noise, interpolation, strain and image contrast on motion measurements, Strain 45 (2) (2009) 160-178.

[58] P. Lava, S. Cooreman, S. Coppieters, M. De Strycker, D. Debruyne, Assessment of measuring errors in DIC using deformation fields generated by plastic FEA, Optics and Lasers in Engineering 47 (7-8) (2009) 747-753.

[59] A. Andrade-Campos, S. Thuillier, P. Pilvin, F. Teixeira-Dias, On the determination of material parameters for internal variable thermoelastic-viscoplastic constitutive models, International Journal of Plasticity 23 (8) (2007) 1349-1379.

[60] T. Furukawa, T. Sugata, S. Yoshimura, M. Hoffman, An automated system for simulation and parameter identification of inelastic constitutive models, Computer Methods in Applied Mechanics and Engineering 191 (21-22) (2002) 2235-2260.

[61] Y. Pannier, S. Avril, R. Rotinat, F. Pierron, Identification of elasto-plastic constitutive parameters from statically undetermined tests using the virtual fields method, Experimental Mechanics 46 (6) (2006) 735-755.

[62] L. Anand, Constitutive equations for the rate-dependent deformation of metals at elevated temperatures, Journal of Engineering Materials and Technology 104 (1) (1982) 12-17.

[63] A. Lush, G. Weber, L. Anand, An implicit time-integration procedure for a set of internal variable constitutive equations for isotropic elasto-viscoplasticity, International Journal of Plasticity 5 (5) (1989) 521-549. 
[64] E. de Souza Neto, D. Perié, D. R. J. Owen, Computational methods for plasticity: Theory and applications, Wiley, 2008.

[65] N. Chawla, Y. L. Shen, X. Deng, E. S. Ege, An evaluation of the lap-shear test for Sn-rich solder/Cu couples: experiments and simulation, Journal of Electronic Materials 33 (12) (2004) $1589-1595$.

[66] V. Sivasubramaniam, M. Galli, J. Cugnoni, J. Janczak-Rusch, J. Botsis, A study of the shear response of a lead-free composite solder by experimental and homogenization techniques, Journal of Electronic Materials 38 (10) (2009) 2122-2131.

[67] Canon, Canon MP-E 65mm f/2.8 1-5x Macro Photo, http://www.canon.co.uk/For_ Home/Product_Finder/Cameras/EF_Lenses/Macro/MP-E_65mm_f2.8_1-5x_Macro_ Photo/.

[68] M. A. Sutton, J. H. Yan, V. Tiwari, H. W. Schreier, J. J. Orteu, The effect of out-of-plane motion on $2 \mathrm{~d}$ and $3 \mathrm{~d}$ digital image correlation measurements, Optics and Lasers in Engineering 46 (10) (2008) 746-757.

[69] T. Guélon, E. Toussaint, J. B. Le Cam, N. Promma, M. Grédiac, A new characterisation method for rubber, Polymer Testing 28 (7) (2009) 715-723.

[70] N. Promma, B. Raka, M. Grédiac, E. Toussaint, J. B. Le Cam, X. Balandraud, F. Hild, Application of the virtual fields method to mechanical characterization of elastomeric materials, International Journal of Solids and Structures 46 (3-4) (2009) 698-715.

[71] S. Avril, P. Badel, A. Duprey, Anisotropic and hyperelastic identification of in vitro human arteries from full-field optical measurements, Journal of Biomechanics 43 (15) (2010) 29782985.

[72] M. Sasso, G. Chiappini, M. Rossi, L. Cortese, E. Mancini, Visco-hyper-pseudo-elastic characterization of a fluoro-silicone rubber, Experimental Mechanics 54 (3) (2014) 315-325.

[73] M. Stein, Large sample properties of simulations using latin hypercube sampling, Technometrics 29 (2) (1987) 143-151. 
[74] D. Müller, G. Hartmann, Identification of materials parameters for inelastic constitutive models using principles of biologic evolution, Journal of Engineering Materials and Technology 111 (3) (1989) 299-305.

[75] R. W. Ogden, G. Saccomandi, I. Sgura, Fitting hyperelastic models to experimental data, Computational Mechanics 34 (6) (2004) 484-502.

[76] X. Yan, G. Li, Study of thermal fatigue lifetime of fan-in package on package (fipop) by finite element analysis, in: International Conference on Electronic Packaging Technology \& High Density Packaging, 2009. ICEPT-HDP’09., IEEE, 2009, pp. 1176-1180.

[77] K. Mysore, G. Subbarayan, V. Gupta, R. Zhang, Constitutive and aging behavior of Sn 3.0 Ag 0. 5Cu solder alloy, IEEE Transactions on Electronics Packaging Manufacturing 32 (4) (2009) 221-232.

[78] M. Motalab, Z. Cai, J. C. Suhling, P. Lall, Determination of Anand constants for SAC solders using stress-strain or creep data, in: 13th IEEE Intersociety Conference on Thermal and Thermomechanical Phenomena in Electronic Systems (ITherm), 2012, IEEE, 2012, pp. 910-922.

[79] R. Rubinstein, S. Atluri, Objectivity of incremental constitutive relations over finite time steps in computational finite deformation analyses, Computer Methods in Applied Mechanics and Engineering 36 (3) (1983) 277-290.

[80] Dassault Systèmes, Abaqus Unified FE software, Personal communication, http://www . 3ds.com/products-services/simulia/portfolio/abaqus/overview/ 